Purdue University Purdue e-Pubs

Open Access Theses

Theses and Dissertations

January 2015

\title{
Hybrid Power Management for Office Equipment
}

Ganesh Gingade

Purdue University

Follow this and additional works at: https://docs.lib.purdue.edu/open_access_theses

\section{Recommended Citation}

Gingade, Ganesh, "Hybrid Power Management for Office Equipment" (2015). Open Access Theses. 1178. https://docs.lib.purdue.edu/open_access_theses/1178

This document has been made available through Purdue e-Pubs, a service of the Purdue University Libraries. Please contact epubs@purdue.edu for additional information. 


\section{PURDUE UNIVERSITY \\ GRADUATE SCHOOL \\ Thesis/Dissertation Acceptance}

This is to certify that the thesis/dissertation prepared

By Ganesh Gingade

Entitled

Hybrid Power Management for Office Equipment

For the degree of Master of Science in Electrical and Computer Engineering

Is approved by the final examining committee:

YUNG-HSIANG LU, Co-Chair

JAN P. ALLEBACH, Co-Chair

JAMES V. KROGMEIER

To the best of my knowledge and as understood by the student in the Thesis/Dissertation Agreement, Publication Delay, and Certification/Disclaimer (Graduate School Form 32), this thesis/dissertation adheres to the provisions of Purdue University's "Policy on Integrity in Research" and the use of copyrighted material.

\section{YUNG-HSIANG LU, Co-Chair}

Approved by Major Professor(s):

Approved by: Michael R. Melloch $05 / 01 / 2015$ 


\title{
HYBRID POWER MANAGEMENT \\ FOR OFFICE EQUIPMENT
}

\author{
A Thesis \\ Submitted to the Faculty \\ of \\ Purdue University \\ by \\ Ganesh P. Gingade \\ In Partial Fulfillment of the \\ Requirements for the Degree \\ of
}

Master of Science in Electrical and Computer Engineering

August 2015

Purdue University

West Lafayette, Indiana 
To Amma, Dad, and Navodaya 


\section{ACKNOWLEDGMENTS}

I would like to thank my advisers, Professor Yung-Hsiang Lu and Professor Jan P. Allebach for their guidance, feedback, and constant support. I learned valuable life lessons in addition to the joy of research. Thanks to Prof.Lu, for introducing me to many other interesting research areas. I am grateful to Hernan Ildefonso Gutierrez and Perry Lea from HP for their constant feedback and suggestions. I would also like to acknowledge Professor James V. Krogmeier for his suggestions.

Thanks to Andrew R. Hughes for providing the printer traces. Special thanks to Wenyi Chen for the implementation and Yufang Sun for the help in setting up the printer. Thanks to High-Efficiency, Low-Power System (HELPS) Group, especially Anup Mohan, Ahmed Kaseb, and Youngsol Koh for all the fun and lively discussions.

My parents are my real source of inspiration and strength. I am eternally grateful for all their sacrifices, patience, and dreams. I am grateful to my brothers - Abhijith and Amith, for their guidance and support. Special thanks to little Smriti for bringing joy to our family, Smitha and Aditi for the support. Thanks to Ajith Karnik, Anirudh HN, Ananda DV, Sumanth Cadambi, Jayaram Mahale, Aravinda J, Ravikumar BT, Navodaya friends for all the fun and good times, MT Karunakaran, Naveen Kumar, Vishrut Vaibhav, Mohit Prakash from Anveshan Telecom for the best experience, and Abhinav Chandrappa, Pramod Magadi, Ravichandra Jagannath, Nishant Swaroop, Mahesh Ramesh, Karthik Kamanna, Suhas Sreehari from Beau Jardin for all the fun.

This research project was sponsored by Hewlett-Packard Company. Their financial support made this work possible. 
TABLE OF CONTENTS

Page

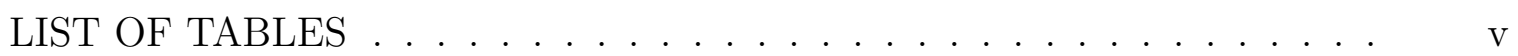

LIST OF FIGURES . . . . . . . . . . . . . . . . . . vi vi

ABSTRACT ........................... ix

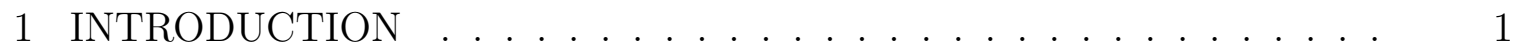

2 RELATED WORK . . . . . . . . . . . . . . . . . 4

2.1 Dynamic Power Management . . . . . . . . . . . . . . . . . 4

2.2 Power Management for Office Equipment . . . . . . . . . . . 5

2.3 Power Policy Selection . . . . . . . . . . . . . . . . . . 6

3 LASER PRINTER SYSTEM . . . . . . . . . . . . . . . 8

3.1 Printer Mechanism . . . . . . . . . . . . . . 8

3.2 Printer Power Cycle . . . . . . . . . . . . . . 10

3.3 Printer Performance Metrics . . . . . . . . . . . . . . . . 11

4 POWER MANAGEMENT POLICY . . . . . . . . . . . . . . . 13

4.1 Printer Activity . . . . . . . . . . . . . . . 13

4.2 Adaptive Multi-Phase Power Management . . . . . . . . . . . 15

4.3 Hybrid Power Management . . . . . . . . . . . . . . . . 24

5 EVALUATION AND RESULTS . . . . . . . . . . . . . . . 28

5.1 Printer Parameter Measurement . . . . . . . . . . . . 28

5.2 Adaptive Multiphase Power Management . . . . . . . . . . . . . . 31

5.3 Hybrid Power Management . . . . . . . . . . . . . . . . . . 32

5.4 Evaluation Window Selection . . . . . . . . . . . . . 40

5.5 Week Analysis . . . . . . . . . . . . . . . . . . 42

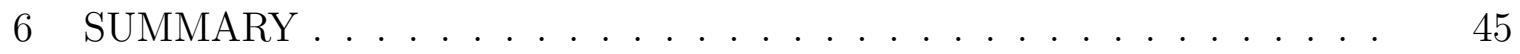






\section{LIST OF TABLES}

Table $\quad$ Page

5.1 Measured printer parameters . . . . . . . . . . . . . . . . . 29

5.2 Quad-phase parameters using pattern search . . . . . . . . . . . 33

5.3 Dual-Phase parameters using pattern search . . . . . . . . . . . . 33

5.4 Average power (Watts) for the candidate power policies. No single policy provides low power for all the printers. The hybrid policy performs better than the best performing policy. . . . . . . . . . . 36

5.5 Average delay (seconds) for the candidate power policies. No single policy provides low delay for all the printers. . . . . . . . . . .

5.6 Average power (Watts) for Printer-2 with the hybrid policy using different evaluation and freeze windows . . . . . . . . . . . . . 38

5.7 Average delay (seconds) for Printer-2 with the hybrid policy using different evaluation and freeze windows . . . . . . . . . . . . . . 38

5.8 Policy switch frequency for Printer-2 with the hybrid policy using different evaluation and freeze windows . . . . . . . . . . . . .

5.9 Average power in Watts for the previous six day schedule. The hybrid policy using a short-term memory of past requests and frequent updates performs better than the policies using scheduling information from the previous six days. . . . . . . . . . . . . . . . .

5.10 Average power in Watts for the previous week schedule. The hybrid policy without using any scheduling information from the past week performs consistently better than the policies using scheduling information. 


\section{LIST OF FIGURES}

Figure

Page

3.1 Color laser printer image formation system. The desired image is formed on a negatively charged photosensitive drum using laser beams. Yellow $(\mathrm{Y})$, magenta $(\mathrm{M})$, cyan $(\mathrm{C})$, and black $(\mathrm{K})$ images are formed on four separate photosensitive drums. The charged toner gets attracted to the drum at the image areas and later transferred onto an intermediate transfer belt (ITB). The toner collected from different colors gets attached to a positively charged paper. Finally the toner is fused and pressed onto a paper to get a permanent image. (Source: HP color LaserJet service manual) . . . . . . . . . . . . . . . . . .

3.2 Printer starts in the idle state, consuming idle power. Upon receiving a request, the printer transitions to a higher power state for serving the request. After serving the request, the printer returns to the ready state and starts a timer. If no request is received within this timeout period, the printer transitions to a low power state. Most of the components are turned off at the low power state. Waking up from the sleep state incurs a significant cost in terms of energy and time. The wakeup energy and delay is printer-specific. . . . . . . . . . . . . . . . . .

4.1 Printer request log of an office printer. Each symbol represents a request. Several attributes are logged at each request like start and end times, job type etc,. Each row belongs to a day of the week plotted over course of 24 hours. . . . . . . . . . . . . . . .

4.2 Cumulative distribution of inter-arrival times for printers placed at different offices. It depicts the ratio of request occurrences with inter-arrival times less than a specific time to the total requests. Break-even time is the time period during which the printer would incur no additional energy irrespective of the power state it stays in after serving a request. . . . .

4.3 Autocorrelation of inter-arrival times (binary) at different lags for printer1. The inter-arrival times are classified using a threshold (break-even time). The inter-arrival times greater than the break-even time are assigned a value '1' else a value ' 0 ' is assigned. The autocorrelation at different lags are computed on the binary sequence to verify any relation among printer requests. The printer requests exhibit a short-term correlation. . . . . . . . . . . . . . . . . . 
Figure $\quad$ Page

4.4 Activity phases ranging from the most busy $\left(\mathrm{AP}_{0}\right)$ to the least busy $\left(\mathrm{AP}_{N-1}\right)$. Timeout $\left(\mathrm{TO}_{k}\right)$ and inter-arrival thresholds $\left(\mathrm{IA}_{k-1, k}, \mathrm{IA}_{k, k+1}\right)$ for an activity phase $\left(\mathrm{AP}_{k}\right)$ are determined using MATLAB's pattern search. The printer in the activity phase $\left(\mathrm{AP}_{k}\right)$ stays in the same phase $\left(\mathrm{AP}_{k}\right)$ or moves to its adjacent activity phase $\left(\mathrm{AP}_{k-1}\right.$ or $\left.\mathrm{AP}_{k+1}\right)$, based on the current inter-arrival time (ia). . . . . . . . . . . . . .

4.5 Computing multiphase parameters using the pattern search optimizer. .

4.6 Sample delay constraints during a weekday for a printer in a general office environment. The constraints are based on the assumption that a printer is used more often during the working hours from 8:00 AM to 6:00 PM. A relaxed constraint is applied during the non-working hours with an intermediate constraint during the transition hours. . . . . . . . . . .

4.7 Hybrid power management consists of a set of candidate power policies and an evaluator. Each candidate policy outperforms the others under different workloads. The candidate policies are evaluated based on a criteria of energy savings or delay during the policy evaluation. The best performing policy becomes active and manages the printer power for the next policy period. . . . . . . . . . . . . . . .

4.8 Hybrid power manager with a policy evaluation window $\left(t_{\text {eval }}\right)$ and freeze window $\left(t_{\text {freeze }}\right)$. The best performing policy during the evaluation window is applied for the next freeze window. The evaluation window can be also set from the previous day or week. . . . . . . . . . . . . . .

5.1 Printer power measurement setup. The power meter and the printer are both connected to a network. A computer is used to control and access both the power meter and the printer. Common unix printing system (CUPS) standard is used to print to a network printer. Test pages are printed and the corresponding power readings are logged. The power meter refreshes the readings after each second. A log file is generated with the time-stamps and power readings. . . . . . . . . . . .

5.2 Printer power measurement reading. A test page is printed while the printer is in (1) ready and (2) sleep state. Power readings are logged at each second. The printer starts in the sleep state consuming a low power. It serves a request consuming power at 650 Watts. The printer enters the ready state waiting for further requests, consuming an average idle power of 70 Watts. It consumes more power while printing from the sleep state than from the ready state. The printer takes more time serving a request from the sleep state. . . . . . . . . . . . . . . . . 
Figure

5.3 Comparison of average power and average delay for different policies. The oracle policy saves the most energy as it knows the future. The factory preset fixed 30 minute timeout keeps the printer awake most of the time and hence less delay per request, but has the power consumption. Immediate shutdown has to wakeup for each request and hence has the delay per request. Multiphase policy balances delay with marginal penalty in power consumption. . . . . . . . . . . . . . . . .

5.4 Histogram of power management policies selected by the hybrid policy. This shows that the best policies varies with the underlying requests. .

5.5 Average power in Watts for Printer-2 with hybrid policy using different evaluation and freeze windows . . . . . . . . . . . . . . 


\begin{abstract}
Gingade, Ganesh Prahlad Rao M.S.E.C.E., Purdue University, August 2015. Hybrid Power Management for Office Equipment. Major Professors: Yung-Hsiang Lu and Jan P. Allebach.

Office machines (such as printers, scanners, fax, and copiers) can consume significant amounts of power. Few studies have been devoted to power management of office equipment. Most office machines have sleep modes to save power. Power management of these machines are usually timeout-based: a machine sleeps after being idle long enough. Setting the timeout duration can be difficult: if it is too long, the machine wastes power during idleness. If it is too short, the machine sleeps too soon and too often - the wakeup delay can significantly degrade productivity. Thus, power management is a tradeoff between saving energy and keeping short response time. Many power management policies have been published and one policy may outperform another in some scenarios. There is no definite conclusion which policy is always better. This thesis describes two methods for office equipment power management. The first method adaptively reduces power based on a constraint of the wakeup delay. The second method is a hybrid with multiple candidate policies and it selects the most appropriate power management policy. Using six months of request traces from 18 different offices, we demonstrate that the hybrid policy outperforms individual policies. We also discover that power management based on business hours does not produce consistent energy savings.
\end{abstract}




\section{INTRODUCTION}

Office equipment may consume significant amounts of energy and the potential of energy savings has not been fully exploited. Today's office equipment is set in "ready" mode to have short response time. However, the machine is idle for most part of business hours [1] and consumes a significant amount of power in the ready mode. As energy efficiency becomes increasingly important, power management is essential for office equipment. The most prevalent power management policy for office equipment is timeout: when the machine becomes idle, a timer is set. While waiting for the timer to expire, the machine stays in the ready mode. If the machine remains idle when the timer expires, the machine enters a lower-power "sleep" mode. The timeout value is set by either the user or the manufacturer in order to comply with regulations for Eco-labels such as Energy Star [2], Blue Angel [3], or Nordic Swan [4]. These regulations stipulate stringent energy requirements for certification (under one Watt after a sufficient period of idle time) [5].

General power management policies employ either predictive or stochastic based approaches [6]. The former predicts the next machine activity based on exponential average, correlation, regression, or heuristics of past observed and predicted idle time [6]. Stochastic techniques model systems as Markov decision processes [7]. Several other policies employ machine learning techniques to learn the power management policy [8], [9], [10]. Some maintain a set of policies and perform online policy selection [11], [12] based on user specified energy-performance criteria. In case of printers, an adaptive timeout policy is proposed [13], [14] using the estimated distribution of past print requests.

Many dynamic power management studies focus on peripheral devices such as hard disk drive (HDD) and wireless local area network (WLAN) controller [7]. Very few studies are devoted to office equipment power management. Office equipment presents 
unique challenges wherein the wakeup energy and delay are significantly higher than the peripheral devices. The idle power of a printer can be 100 Watts, while a HDD's idle power is around 5 Watts [12]. The wakeup energy for a printer can be 2000 Joules compared to a HDD's 20 Joules. The printer request is highly dynamic and depends on the location and time. Office equipment encounters a variety of request scenarios based on the offices' functions (business office, academic units, or student laboratories) or the time of the request (day, night, weekday, or weekend). Best power management policy for office equipment depends on hardware and workload. For frequent requests, a preferred power management policy would have a long timeout to prevent the printer going to sleep. For sparse requests, a better policy would shutdown soon after each service. Hence a fixed timeout cannot always achieve desirable energy savings. Some printers provide options to set custom schedules for sleep and wakeup times for each day of the week or holidays.

Using six months of request traces from 18 different printers deployed at Purdue University, we conclude that fixed timeout or preset schedules cannot achieve consistent energy savings for printers in different offices. We propose two policies for printer power management. The first policy divides the printer workload into distinct phases and provides a timeout for each phase. The timeouts are determined from the printer past request based on a constraint of the wakeup delay. The second selects the best power policy from a set of candidate policies based on the observed request pattern. We evaluate this hybrid policy and demonstrate that it is better than the individual policies. We also show that, contrary to intuition, a policy with scheduling information (such as weekdays vs. weekends) underperforms the policies without such knowledge about business hours.

This thesis has the following contributions:

1. We propose a power management policy with multiple timeouts, each determined from the printer's past requests, balancing power and delay. 
2. We demonstrate that no single power management policy performs consistently for different printers in different offices.

3. We propose a hybrid power policy that outperforms individual power management policies.

4. We demonstrate that the hybrid power policy performs best with frequent policy updates and using short-term memory of past printer requests.

5. We examine the policy with scheduling information (such as weekdays vs. weekends) and show that it does not produce consistent power savings. 


\section{RELATED WORK}

Related work in power management can be found for peripheral devices like hard disk drives, displays, and wireless local area network (WLAN) controller [7]. Few studies have been devoted exclusively for office equipment. In the following sections, we discuss the related work and compare our policies with the existing policies.

\subsection{Dynamic Power Management}

Dynamic power management policies can be broadly classified as timeout based, predictive, or stochastic [15], [6], [16]. In timeout based policies, an idle machine is set to a low power state after the expiry of a timeout period. The timeout could be pre-configured to a fixed value based on expected future requests or changed dynamically. The value could be statically or adaptively determined [17]. In a static timeout policy, a pre-configured value is used irrespective of the request conditions. In an adaptive timeout policy, the value is changed according to the workload changes. Ramanathan and Gupta [18] describe an adaptive timeout policy based on a device's break-even time. Break-even time is the time period during which the device would incur no additional energy irrespective of the energy state it stays in after serving a request. Shih and Wang [19] present a policy for mobile devices that can adapt to self-similar workloads [32] exhibited by human interactions. Guo et al. [31] take into account human behavior aspects to determine the best policy for the adaptive energy management. Prediction based policies rely on past request patterns to predict a device's future request. The prediction-based policies attempt to predict future requests and set the device's power state. The prediction of inter-arrival times is based on the observed patterns: exponential average, correlation, regression, or heuristics of past observed and predicted idle times [7], [6], [20], [21]. For example, future inter- 
arrival times are predicted using an observed pattern like L-Shape [22], where it is assumed that a short busy period is followed by a long idle period. Stochastic policies model systems as Markov decision processes. Both Markov and Semi-Markov models are considered. The policies are considered constrained optimization problems [7]. The models trade off between power and latency. They attempt to estimate the underlying request arrival distribution and globally optimize the expected power and latency. Both stationary and non-stationary requests are considered [23]. Discrete and continuous time stochastic models have been proposed [24], [7]. Several other policies employ machine learning techniques [25] to learn the power management policy from the device environment. Supervised learning and reinforcement learning have been explored [8], [9], [10], [26], [27], [28].

The competitive analysis technique can be used to compare online policies [29]. In this approach the performance of an online policy is compared against an optimal offline oracle policy. The policy is c-competitive if for any sequence of requests, its worst case performance is bounded by c times the performance of the offline oracle policy. The fixed timeout policy has been shown to be a 2-competitive [18], if the timeout is set to the break-even time. The worst case power dissipation expected from a fixed timeout policy is twice the oracle's power. The dual-timeout adaptive policy [18] is 3-competitive, but shown to achieve better performance in practice. The competitive ratio's low bound is 1.58 [29] for the best timeout policy. User annoyance is attributed to latency during system power management [17]. Irani and Pruhs [30] mention a need for a policy which allows a user or system to preferentially choose between optimizing one resource over another (for example, optimizing power based on a delay constraint).

\subsection{Power Management for Office Equipment}

The most prevalent power management policy for office equipment is timeout. The timeout value is set by either the user or the manufacturer in order to comply 
with regulations for Eco-labels. Larson [33] describes a policy of selecting power

state for a peripheral by examining the activity packets. Ciriza et al. [13] describe a timeout based policy, wherein a fixed timeout is estimated to minimize a cost function. The cost function is based on the distribution of the past (week or month) observed inter-arrival times and penalty (wake-up delay and energy). Durand et al. [14], [34] describe an adaptive timeout policy using a hidden Markov chain. These studies suggest using long-term observations to improve energy savings. In contrast, we show that the power management policy performs better using short-term past requests.

\subsection{Power Policy Selection}

Pettis and $\mathrm{Lu}$ [12] introduce power policy selection in an operating system and show one policy outperforming another under some conditions. It may be difficult, or even impossible, to design the "best" policy for all conditions. A software framework called the Homogeneous Architecture for Power Policy Integration (HAPPI) is defined for system power management. The framework selects an active power management policy by choosing the best estimate for an evaluation metric, such as total energy consumption or energy-delay product. The evaluation is performed every 20 seconds and the active policy is selected. An experimental setup is demonstrated that selecting policies can achieve better reduction in system energy. Different workloads are considered to demonstrate the notion of "no one policy fits all". Helmbold et al. [35] maintain a set of "expert" policies and use multiplicative weights to make timeout predictions. Dhiman and Rosing [11] describe an online learning policy to select among a set of possible policies and voltage-frequency settings. The online learning policy maintains a set of "experts" and select an expert that has the best chance to perform well based on the characterization of the current workload. An evaluation is performed at the end of each idle period to select among the experts. It demonstrates that the performance is at least as good as the best expert across different workloads. In addition to using the policy selection as described in the above 
two papers, we propose how to perform policy evaluation and determine the period for better results. We also show that better performance is achieved using short-term past requests. 


\section{LASER PRINTER SYSTEM}

\subsection{Printer Mechanism}

Office imaging equipment includes the following product types: printers, scanners, copiers, and facsimile machines. In this chapter, we discuss laser printers with a focus on the printer mechanism and performance metrics. The discussion applies to other office equipment. Laser printers produce high quality text and graphics on plain paper. Laser printers have mechanical and thermal components [36] which affect power management. Laser printers use electrically charged rotating drums. A laser beam is used to alter the charge components of the drum. Dry ink or toner is attracted to the charge altered areas of the drum. The toner is subsequently transferred to a paper and fused using heat and pressure. As shown in Fig 3.1, the laser printer imageformation system consists of the following parts: laser for scanning, print cartridge, imaging drums, intermediate transfer belt (ITB) and fuser. The process of image formation onto a paper involves the following steps [36]: (i) latent image formation, (ii) development, (iii) transfer, (iv) fusing.

Once the printer gets the data, a latent image is formed on the surface of the

photosensitive drum. To form the desired latent image, the photosensitive drums are initially stripped of any residual charges by exposing them to LED light. A charging roller is used to negatively charge the drum. A laser beam is used to strike the surface of the photosensitive drum at those areas where an image is desired. The image areas on the drum acquire neutral charge and are ready to accept the toner (dry ink). Next, the toner is given negative charges during the development. The toner is placed in those areas neutralized by the laser beam. The areas not struck by the laser beam still retain negative charges and thus repel the negatively charged toner. An image is formed on the photosensitive drum. The image is transferred 


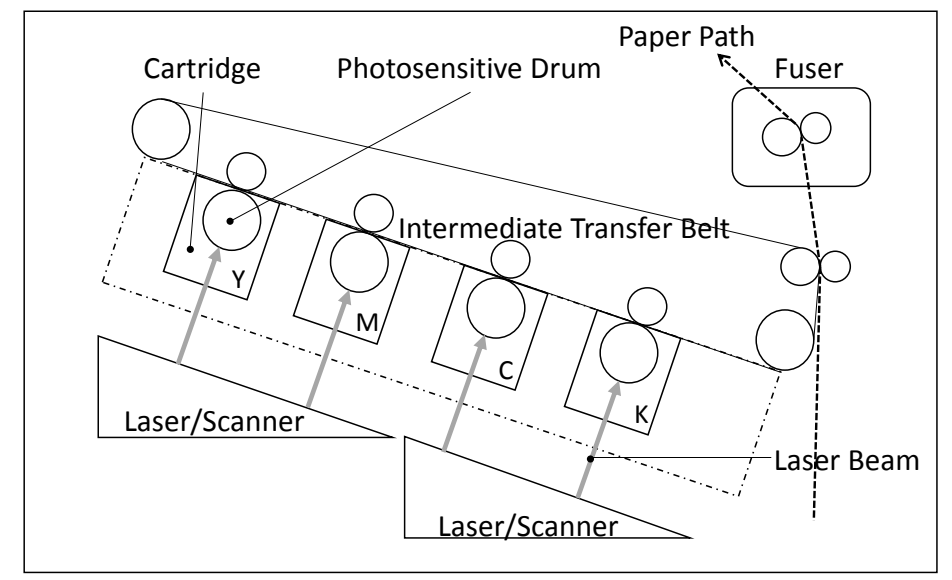

Fig. 3.1. Color laser printer image formation system. The desired image is formed on a negatively charged photosensitive drum using laser beams. Yellow $(\mathrm{Y})$, magenta $(\mathrm{M})$, cyan $(\mathrm{C})$, and black $(\mathrm{K})$ images are formed on four separate photosensitive drums. The charged toner gets attracted to the drum at the image areas and later transferred onto an intermediate transfer belt (ITB). The toner collected from different colors gets attached to a positively charged paper. Finally the toner is fused and pressed onto a paper to get a permanent image. (Source: HP color LaserJet service manual) 
onto a paper using the ITB. At first, the ITB is given a positive charge. As the ITB contacts the photosensitive drum, the negatively charged toner gets attracted to the ITB. Likewise, toners of different colors: yellow, magenta, cyan, and black are transferred onto the ITB in sequence. The paper is given a positive charge. Thus on contact with the ITB, the negatively charged toner on the ITB gets attracted to the positively charged paper. The complete toner image gets transferred onto the paper. The paper is then passed through the heated and pressurized rollers to melt the toner and get the permanent image. The fuser accounts for the highest power usage in a laser printer. The fuser has to be at the right temperature before the printing can begin. The primary power saving feature available in most the laser printers is to turn off the fuser. It takes a considerable amount of time (around 10 seconds) before the fuser returns to the required temperature after being turned off. Thus any power management policy has to factor in the fuser property to balance power savings and delays.

\subsection{Printer Power Cycle}

The printer cycles through the following sequence each time there is a request as illustrated in Fig 3.2. The printer at the sleep state enters a waiting period upon a request and starts heating the fuser. This takes a considerable amount of time. Upon heating the fuser to the right temperature, the printer enters the ready state. During the printing process, an image is formed on the photosensitive drum by striking a laser beam which is eventually transferred using a charged toner onto a paper and fused. The printer then enters the standby state wherein the fuser is kept ready by constantly applying heat. After a specified length of inactivity, the printer enters the sleep state as a power saving feature. During the sleep state, energy is conserved by turning off many components, in particular the fuser. Some printers have multiple sleep states and different sets of components are turned off. For example, the printer 
HP Color LaserJet CM3530 [36] has the following power-saving modes: (i) power off, (ii) ready, (iii) shallow suspend, (iv) almost one Watt suspend, (v) deep suspend.

At each power state, a specific set of components is kept on with the rest turned off. Different printer manufacturers select different sets of components to achieve desired energy conservation and swift response. Fig 3.2 shows a printer with multiple power states. There is an associated power dissipation at each state. The sleep state dissipates the lowest power. There is a cost in terms of wakeup energy which is expended whenever the printer transitions from a low power state to a higher state. Also there is a considerable latency involved in the transition. To conserve power, the printer has to transition to a low power state. As the printer transitions to a low power state, it also incurs a cost in terms of energy and latency, while waking up to a request. Power management intends to conserve power with little wakeup delay (user experience impact).

\subsection{Printer Performance Metrics}

Printer performance is measured by the number of pages per minute (PPM). Each printer is classified based on PPM and has to meet a specific set of power regulations. Office equipment has to comply with regulations for Eco-labels such as Energy Star [2], Blue Angel [3], or Nordic Swan [4]. These regulations stipulate stringent energy requirements for certification (under one Watt after a sufficient period of idle time) [5]. Only top 25 percent of the printers meeting the requirements are certified. For a printer, the performance metrics include Sleep-to-First-Page-Out and Sleep-to-FirstCopy-Out. The latest printers can achieve sleep to first page out of under 5 seconds from less than 5 Watt. Most printers have sleep to first page out in the range of 12 to 20 seconds [37]. Users often put higher priority on the performance (less than 10 seconds delay from low power mode) than energy efficiency and feel inconvenienced while waiting for the printer to wake up. Energy efficiency and high performance are usually conflicting goals. To improve energy efficiency, a printer should sleep 


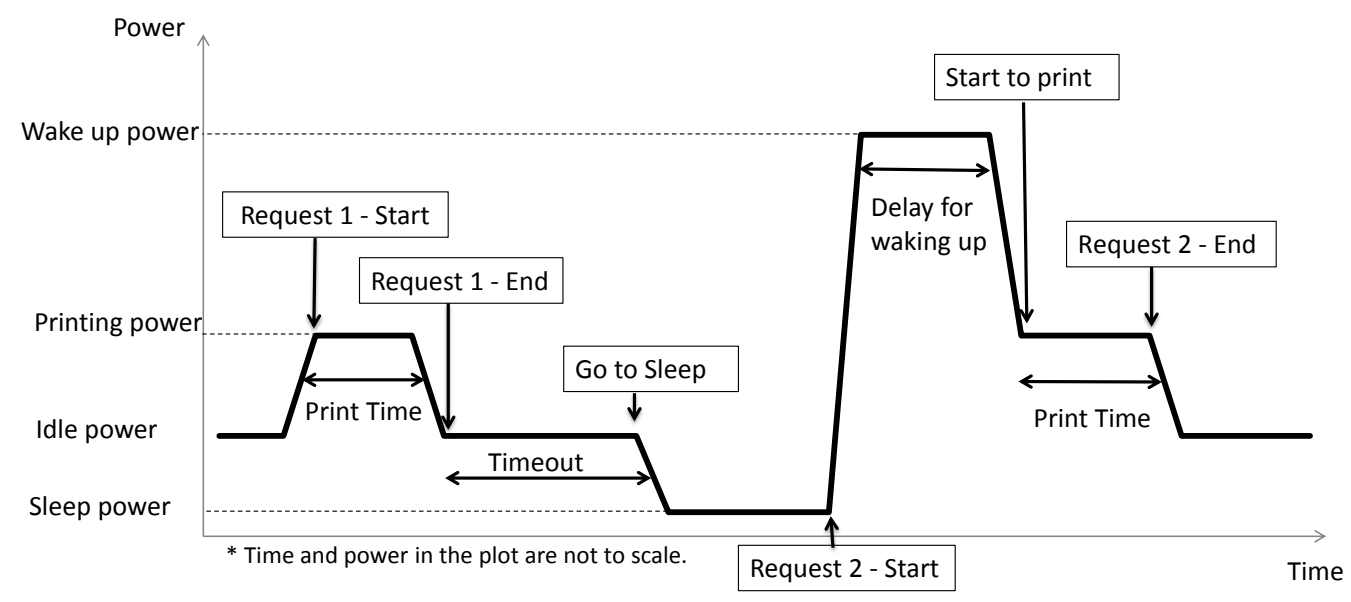

Fig. 3.2. Printer starts in the idle state, consuming idle power. Upon receiving a request, the printer transitions to a higher power state for serving the request. After serving the request, the printer returns to the ready state and starts a timer. If no request is received within this timeout period, the printer transitions to a low power state. Most of the components are turned off at the low power state. Waking up from the sleep state incurs a significant cost in terms of energy and time. The wakeup energy and delay is printer-specific.

often. To improve performance, a printer should stay ready. There have been recent improvements in fuser heating technology and engine mechanical recovery, which constitute the main bottleneck in the printer's response time from sleep. Nevertheless, the delay due to wakeup is still noticeable. Better performance can be achieved with an intelligent power manager inside the printer determining when to sleep. Thus it is desirable to achieve more energy savings with less user inconvenience by balancing between the energy efficiency and performance. 


\section{POWER MANAGEMENT POLICY}

Chapter 2 describes the existing studies on power management policies. Most of the studies focus on peripheral devices and very few are devoted to printer power management. Chapter 3 describes the printer system and unique challenges associated with printer power management. The printers have significantly higher wakeup energy and delay compared with the peripheral devices. We measure the wakeup energy and delay for a commercial printer in Chapter 5. Stringent governmental regulations on power conservation conflicts with the user demand for a swift response. Hence a better power management policy for printers is desirable. We study the request traces from printers installed at 18 different offices at Purdue University in search for a better power management policy for printers. We demonstrate two power management policies specific to printers: (1) Adaptive multiphase power management and (2) Hybrid power management. Adaptive multiphase power management relies on the past printer requests to classify the workload and derive timeouts for the future. The hybrid power management performs policy selection, given a set of candidate power management policies. In Chapter 5, we perform evaluations and compare our new power management policies with the existing policies.

\subsection{Printer Activity}

As part of the solution towards better printer power management, six months of request traces from printers at 18 different offices were studied. The printers were located at different offices like business office, administrative office, faculty room, and student lab. The traces contained the sequences of timestamps corresponding to the printer requests like print, scan, fax, and copy. The timestamps were logged at a resolution of one second for each of the request's start and end time. Figure 4.1 
shows the request trace of an office printer over a month. Each symbol corresponds to a printer request. Each row belongs to a day of the week plotted over a course of 24 hours. We can see most requests during the weekdays in contrast to limited requests during weekends. Similarly, the requests are more frequent during the business hours starting from 8:00 AM to 6:00 PM compared with non-business hours. Similar patterns of requests were observed for most other office printers. Fig 4.2 depicts the cumulative distribution of inter-arrival times for printers placed at five different office rooms over six months. It can be observed that the request patterns are unique to the office printers. Some printers have more frequent usage than the others. For example, printer-3 has the most frequent usage with half of its inter-arrival times less than 3 minutes. While printer-1 has half of its inter-arrival times less than 15 minutes.

The printers are idle most of the time with $20 \%$ of the requests arriving no sooner than 30 minutes. Usually the printers are set in ready mode to serve a request immediately. If the printers are idle most of the time, they waste considerable amounts of energy. From Fig 4.2, we also see a burst request among printers with $40 \%$ of inter-arrival times less than 5 minutes. Setting the printers to low power states would seriously degrade performance as printers would take considerable amounts of time waking up. From Fig 4.3, we see that there is a short-term correlation among printer requests. We compute the autocorrelation on the binary inter-arrival time sequence to verify any relation among printer requests. The binary sequence is computed using a threshold (break-even time). Any inter-arrival time greater than the break-even time is assigned '1' else a '0' is assigned. In summary, printers are idle most of the time with burst of requests in between. The printer requests exhibit a short-term correlation. We use these properties to devise new power management policies specific to printers in the rest of this chapter. 


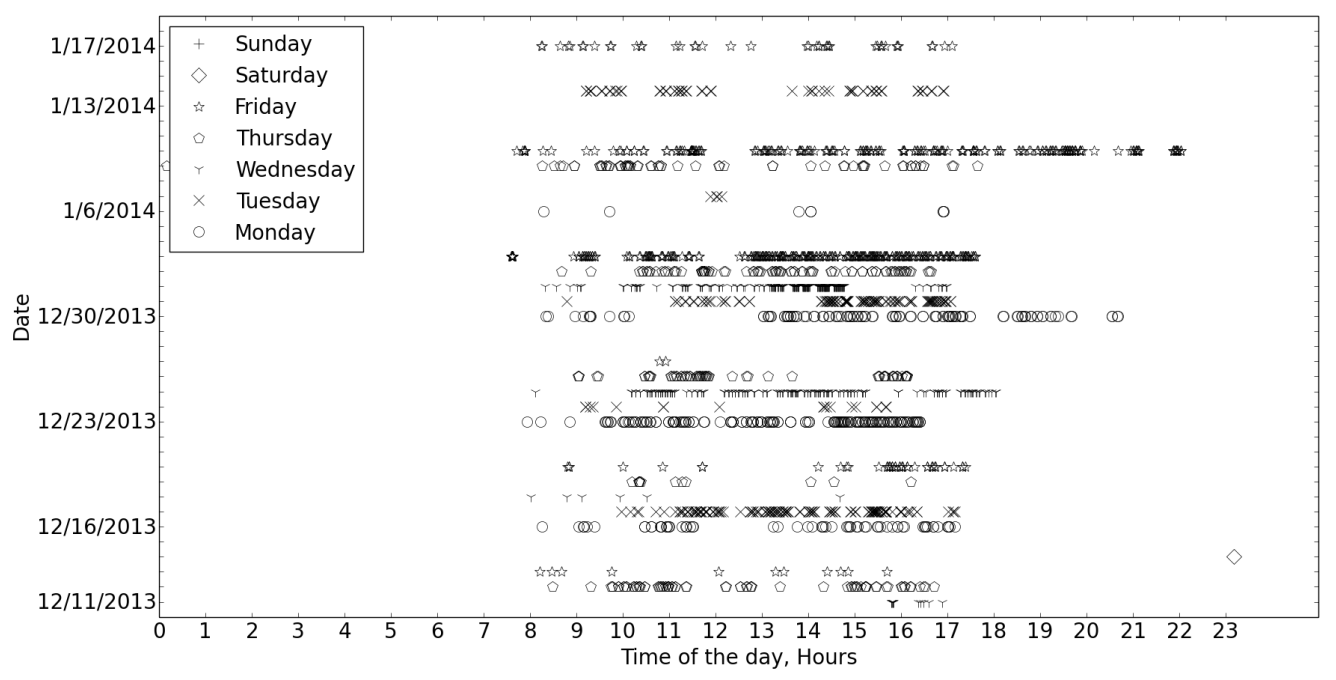

Fig. 4.1. Printer request log of an office printer. Each symbol represents a request. Several attributes are logged at each request like start and end times, job type etc,. Each row belongs to a day of the week plotted over course of 24 hours.

\subsection{Adaptive Multi-Phase Power Management}

From the previous section, we observe the following: (1) The printer requests are unique to the offices. (2) The printer requests exhibit a unique pattern over a day and week. (3) The printer is idle most of the time with burst of requests in between. (4) There is a short-term correlation among the printer requests. (5) Timeout is the most prevalent power saving feature among the printers. The existing single timeout policy is ineffective for a variety of printer workloads. Although a sleep and wakeup schedule is provided for the printer, it is manual and does not accurately characterize the printer workload. Hence there is a scope for better power management policy for the printers, that characterizes the printers' workload and sets the appropriate power states to balance power and delay. We propose an adaptive multiphase policy that compartmentalizes the printer requests into multiple activity phases ranging from 


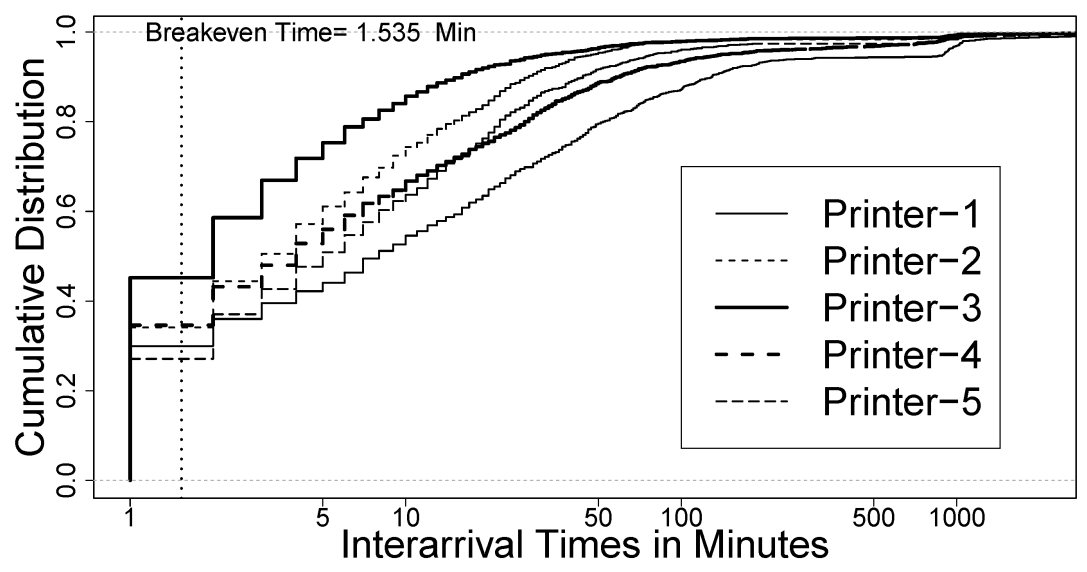

Fig. 4.2. Cumulative distribution of inter-arrival times for printers placed at different offices. It depicts the ratio of request occurrences with inter-arrival times less than a specific time to the total requests. Break-even time is the time period during which the printer would incur no additional energy irrespective of the power state it stays in after serving a request.

the most busy to the least busy. The activity phases are configurable to reflect the variations in the printer workload. For example, during the non-business hours with less variations in the printer requests, we can use dual activity phases. With more variations in the printer requests during the business hours, more number of phases can be used. Each activity phase reflects a printer workload and hence a timeout could be used to set an appropriate power state. The busier the activity phase is, the longer the timeout is set and vice versa. In a busier phase, the printer requests are more frequent and hence a longer timeout would prevent frequent shutdowns, thus providing swift response. Similarly, in a lesser busy phase, a shorter timeout would shutdown the printer more often and save power. Thus based upon the underlying requests, an appropriate activity phase is entered and a corresponding timeout is set. We restrict the phase transitions to adjacent phases to account for short-term correlation found among the printer requests. The phase transitions are based on the request inter-arrival times and a set of thresholds at each phase. The printer 




Fig. 4.3. Autocorrelation of inter-arrival times (binary) at different lags for printer-1. The inter-arrival times are classified using a threshold (break-even time). The inter-arrival times greater than the breakeven time are assigned a value ' 1 ' else a value ' 0 ' is assigned. The autocorrelation at different lags are computed on the binary sequence to verify any relation among printer requests. The printer requests exhibit a short-term correlation.

would incrementally transition to finally reach an activity phase corresponding to the underlying workload. For example during a typical weekday, the printer would start in the least busy phase with the shortest timeout. During business hours, the printer would incrementally transition to a more busy phase depending upon the workload and transition back to the least busy phase at the end of the business hours. The parameters to switch the activity phase and the corresponding timeouts are computed from the past requests. The parameters are updated periodically to reflect the changes in the printer workload.

We illustrate the multiphase policy using the following example. Consider a printer with the break-even time of one minute and a wakeup delay of 10 seconds. Consider a case where all the requests arrive too soon, for example inter-arrival times less than one minute. We can use a single-phase policy with a timeout of greater than one minute. The printer is thus ready for all the requests and never goes to sleep. This 
single-phase policy provides the best energy savings and delay. Similarly, a singlephase policy with an immediate shutdown provides the best energy savings for the case where every requests arrive late, for example inter-arrival times greater than one hour. In this case, the average delay is 10 seconds as the printer shuts down at the end of each request. For the case with bursty requests followed by long idle periods, for example with $90 \%$ of the inter-arrival times less than one minute and the rest greater than one hour, the best energy savings and delay is achieved using a dual-phase policy with the timeouts of one minute and immediate shutdown (zero minute), and an inter-arrival threshold of one minute. The policy shuts down the printer for $10 \%$ of the requests providing an average delay of one second. Using a single policy the printer is idle until the timeout, even when the requests arrive late, wasting energy. For another case with $50 \%$ of the inter-arrival times less than five minutes, $90 \%$ less than 15 minutes and the rest greater than one hour, using a dual-phase policy with the timeouts of 15 minutes and immediate shutdown, we can achieve an average delay of one second, however there is a degradation in the energy savings as the printer is idle for most of the time. We can use a relaxed delay constraint of five seconds and search for the timeouts and inter-arrival threshold for the dual-phase that shuts down the printer for $50 \%$ of the time. We use the timeouts of five minutes and immediate shutdown, and an inter-arrival threshold of five minutes to achieve a delay constraint of less than five seconds with the energy savings improvement. The dual-phase policy shuts down the printer immediately for inter-arrival times greater than five minutes and keeps the printer ready otherwise. Thus the printer wakes up for $50 \%$ of the total requests providing an average delay of five seconds. Thus, we search for the timeouts and inter-arrival thresholds based on the requests and an input delay constraint.

Fig 4.4 describes a general N-phase power management policy, with the activity phases ranging from the most busy $\left(A P_{0}\right)$ to the least busy $\left(A P_{N-1}\right)$. Each activity phase $\left(A P_{k}\right)$ has an associated timeout $\left(T O_{k}\right)$ and inter-arrival thresholds $\left(I A_{k-1, k}, I A_{k, k+1}\right)$. The printer in an activity phase $\left(A P_{k}\right)$ stays in the same phase $\left(A P_{k}\right)$ or transitions to its adjacent phases $\left(A P_{k-1}\right.$ or $\left.A P_{k+1}\right)$ based upon the current 


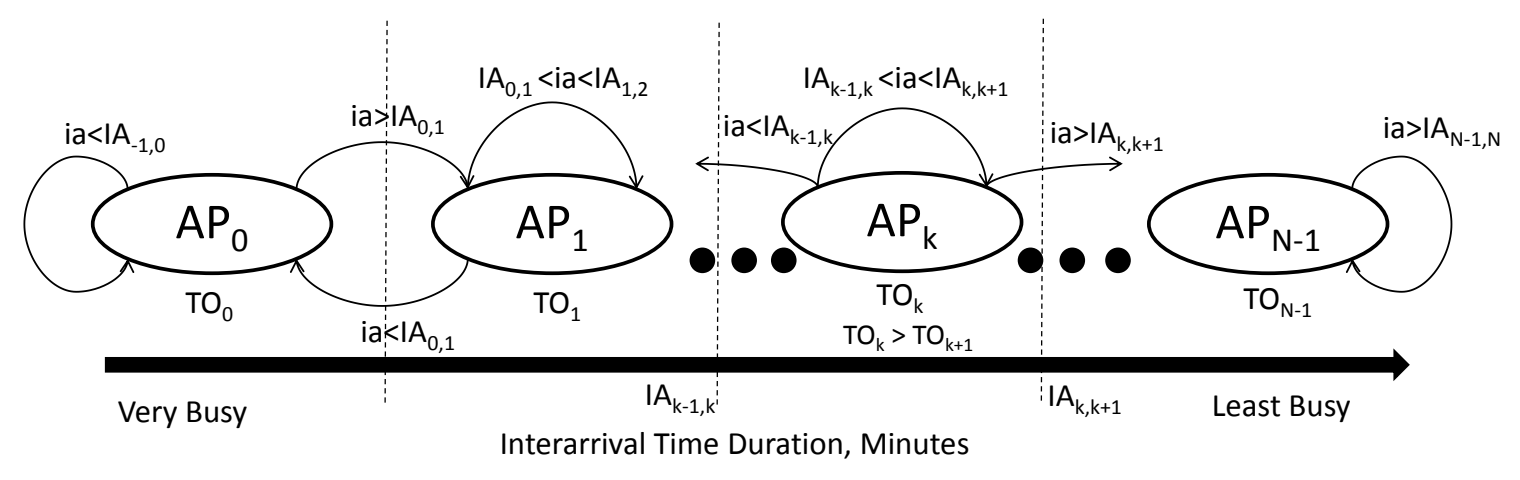

Fig. 4.4. Activity phases ranging from the most busy $\left(\mathrm{AP}_{0}\right)$ to the least busy $\left(\mathrm{AP}_{N-1}\right)$. Timeout $\left(\mathrm{TO}_{k}\right)$ and inter-arrival thresholds $\left(\mathrm{IA}_{k-1, k}, \mathrm{IA}_{k, k+1}\right)$ for an activity phase $\left(\mathrm{AP}_{k}\right)$ are determined using MATLAB's pattern search. The printer in the activity phase $\left(\mathrm{AP}_{k}\right)$ stays in the same phase $\left(\mathrm{AP}_{k}\right)$ or moves to its adjacent activity phase $\left(\mathrm{AP}_{k-1}\right.$ or $\left.\mathrm{AP}_{k+1}\right)$, based on the current inter-arrival time (ia). 
inter-arrival time $(i a)$. The number of phases, $\mathrm{N}$ is configurable and can be set based on the variations in the workload. The timeout $\left(T O_{k}\right)$ and the inter-arrival thresholds $\left(I A_{k-1, k}, I A_{k, k+1}\right)$ are computed from the past printer requests and updated periodically. At each request, the inter-arrival time $(i a)$ is computed as the difference in the time between the current request and the past request instance. The activity phase $\left(A P_{k}\right)$ is updated based on the current inter-arrival time $(i a)$. The printer at the activity phase $\left(A P_{k}\right)$ moves to a more busy phase $\left(A P_{k-1}\right)$ for $i a$ less than the inter-arrival threshold $I A_{k-1, k}$. It moves to a lesser busy phase $A P_{k+1}$ for $i$ a greater than the threshold $I A_{k, k+1}$. It stays in the same phase otherwise. The timeout corresponding to the new activity phase is applied for the next printer idle period, until the next request arrives. The multiphase parameters are updated periodically (every day, week, etc) using the past requests to reflect any changes in the printer workload. Thus, given a set of requests and the printer model, the goal is to compute the parameters for an N-phase power management policy: $T O_{k}, I A_{k-1, k}$, and $I A_{k, k+1}$ for $0<k<N-1$. The parameters should provide the best energy savings with swift response. However, the power and delay are dual in nature. Achieving a low power would incur a cost on delay and vice versa. Hence, we search for parameters that provide the best energy savings under a given delay constraint (for example less than 3 seconds).

We employ optimization methods to search for the multiphase parameters under a given delay constraint. Since the printer is not continuous with power state transitions (active to sleep, etc), we look for the optimization methods that do not use derivatives. Pattern search [38] is one such optimization method that does not use derivatives. Fig 4.5 describes a method for searching the multiphase parameters $\left(T O_{k}, I A_{k-1, k}\right.$, and $\left.I A_{k, k+1}\right)$ using the pattern search optimizer. We use the pattern search optimizer in MATLAB [39] to find the multiphase parameters. We use a printer model with measured parameters (wakeup delay, wakeup energy, active power, and sleep power) that returns an average power for a given set of candidate multiphase parameters and the delay constraint. The model returns a very high power, if the delay constraint is 
not met. The pattern search uses the known request instances and the printer model to find the best parameters. The pattern search converges onto the best parameters that provide a low power under the given delay constraint in finite time. In order to prevent false convergence, the pattern search optimization is performed multiple times with random initial parameters.

The pattern search [38] uses derivative free heuristic methods to search for the best parameters. The pattern search can be illustrated using the following example of a compass search: given a function with two parameters, the pattern search starts with an initial step size and reduces it iteratively, until a threshold is reached. It evaluates the function at each set of candidate parameters along the north, south, east, and west by incrementing or decrementing the parameters by the step size. The candidate parameters that provide a reduction in function is selected for the next iteration. If no reduction is achieved along any direction, the step size is reduced by half and the search is continued. The pattern search is terminated when the step size reaches a lower threshold.

Fig 4.6 describes an example of the multiphase power management for a printer in a general office environment. During the non-working hours (before 6:00 AM and after 7:00 PM) a dual activity phase is used with a relaxed delay constraint of less than 5 seconds. During the work transition hours (6:00 AM - 8:00 AM, Lunch hour, and 6:00 PM - 7:00 PM) a slightly tighter constraint of 4 seconds is used. Finally, during the working hours four activity phases with a constraint of less than 2.5 seconds is used.

In summary, the multiphase power management policy compartmentalizes the printer workload into multiple phases and provides a set of parameters that can be used to reach the relevant phase from any phase. The policy would transition to a relevant phase, based on the current workload and an appropriate timeout is applied balancing power and delay. 


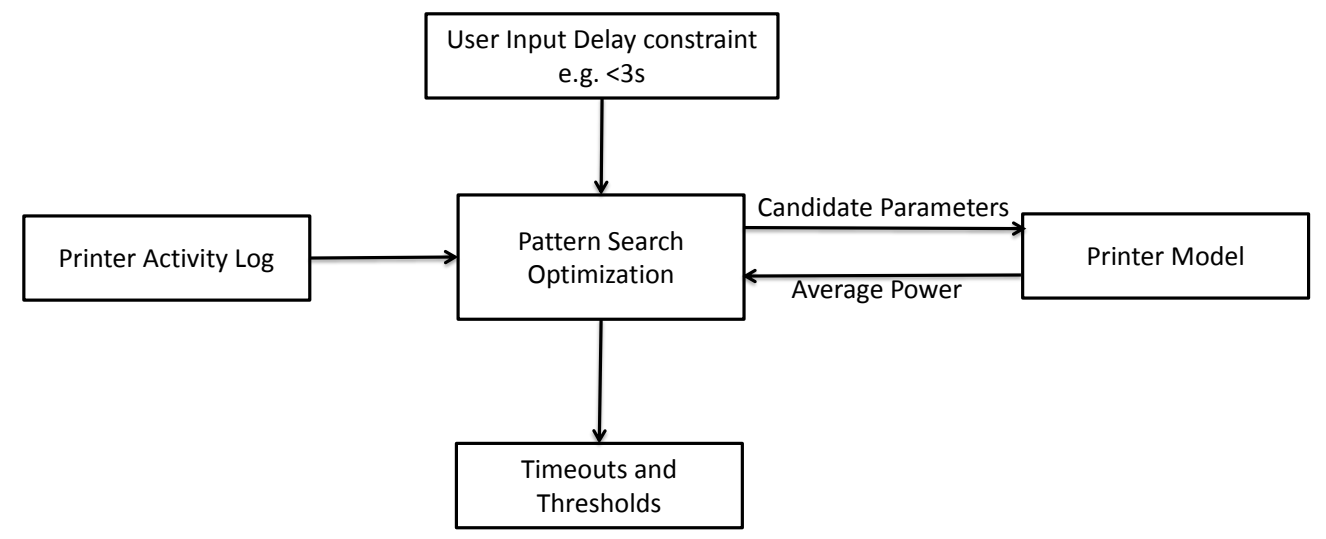

Fig. 4.5. Computing multiphase parameters using the pattern search optimizer. 


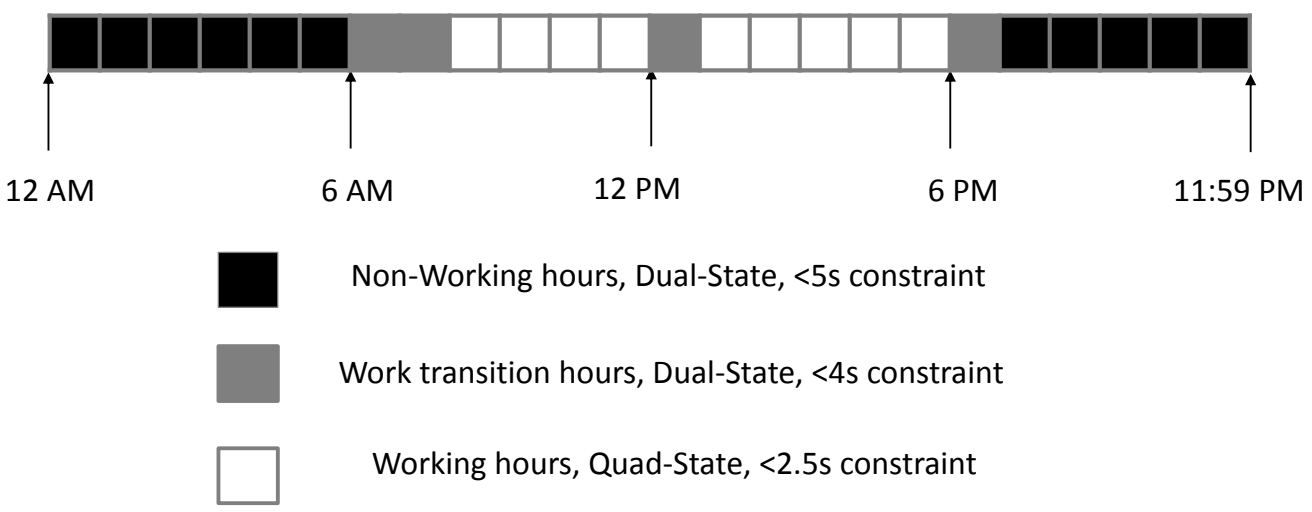

Fig. 4.6. Sample delay constraints during a weekday for a printer in a general office environment. The constraints are based on the assumption that a printer is used more often during the working hours from 8:00 AM to 6:00 PM. A relaxed constraint is applied during the non-working hours with an intermediate constraint during the transition hours. 


\subsection{Hybrid Power Management}

In the previous section, we described a power management policy that compartmentalizes the printer requests into multiple phases. Based on the current workload, the printer transitions to a relevant phase and an appropriate timeout is applied. The multiphase policy is based on the following observations: (1) The printer requests exhibit a unique pattern across a day and week. (2) There is a short-term correlation among the printer requests. (3) Timeout is available for the power management. The multiphase policy provides the following features: (1) The activity phases are configurable to accommodate the workload variations. For example, dual-phase or quad-phase depending upon the expected workload (2) The policy could be scheduled based on the preset hours to accommodate the request pattern observed among the printers. For example, a dual-phase with a relaxed delay constraint during the nonbusiness hours and a quad phase with a tight delay constraint during the business hours. (3) The multiphase parameters are computed from the past requests to adapt to the changes in the workload. However, the multiphase policy has the following drawbacks: (1) The compartmentalization is performed using parameters computed from the past requests and does not reflect the current workload. There is no conclusive evidence of a long-term correlation among the requests, although we observe a short-term correlation.(2) Upon reaching an activity phase, a single timeout is applied irrespective of the workload.

Based on the shortcomings of the multiphase policy, we propose a second policy hybrid policy that attempts to characterize the current workload and selects a policy instead of a timeout. Essentially, power management is a problem of workload characterization. A policy that accurately characterizes the underlying workload is able to successfully perform power management achieving energy savings with acceptable delay. We show in a later chapter by considering different policies that each policy performs well for a different workload. We evaluate each of the policy's performance (energy savings) on multiple printer traces from different offices. We evaluate the 
policy's overall performance as well as the performance during a short time period. We can associate a workload with a policy and hence, with a combination of power management policies, we can achieve a better performance. The hybrid policy is thus a combination of individual candidate policies that achieves better performance overall than a single policy. Instead of explicitly characterizing a printer's workload as a single pattern, we select a policy associated with the workload. As the workload changes, we select a different policy.

Fig 4.7 describes the hybrid policy that performs the policy selection. The hybrid policy selects and applies policies regularly. The hybrid policy consists of the following components: (1) Candidate power management policies (2) An evaluator that performs the policy evaluation based on a criteria (3) The printer. The goal is to characterize the current printer workload and to select the best power policy. We perform policy selection periodically to reflect changes in the workload. Assuming the workload does not change drastically, we rely on a policy's performance during the immediate past to select it for the future. As shown in Fig 4.8, at current time $t_{\text {current }}$, an evaluation is performed on the just concluded workload. An evaluation window of time period $t_{\text {eval }}$ is used to find a policy that performed best during the evaluation window. Assuming the workload does not vary drastically, the best performing policy would also perform better for the future workload. The best performing policy is retained for the next freeze period $t_{\text {freeze }}$. The hybrid power management performs the workload characterization and in turn provides the best performance overall as compared with a single policy. 


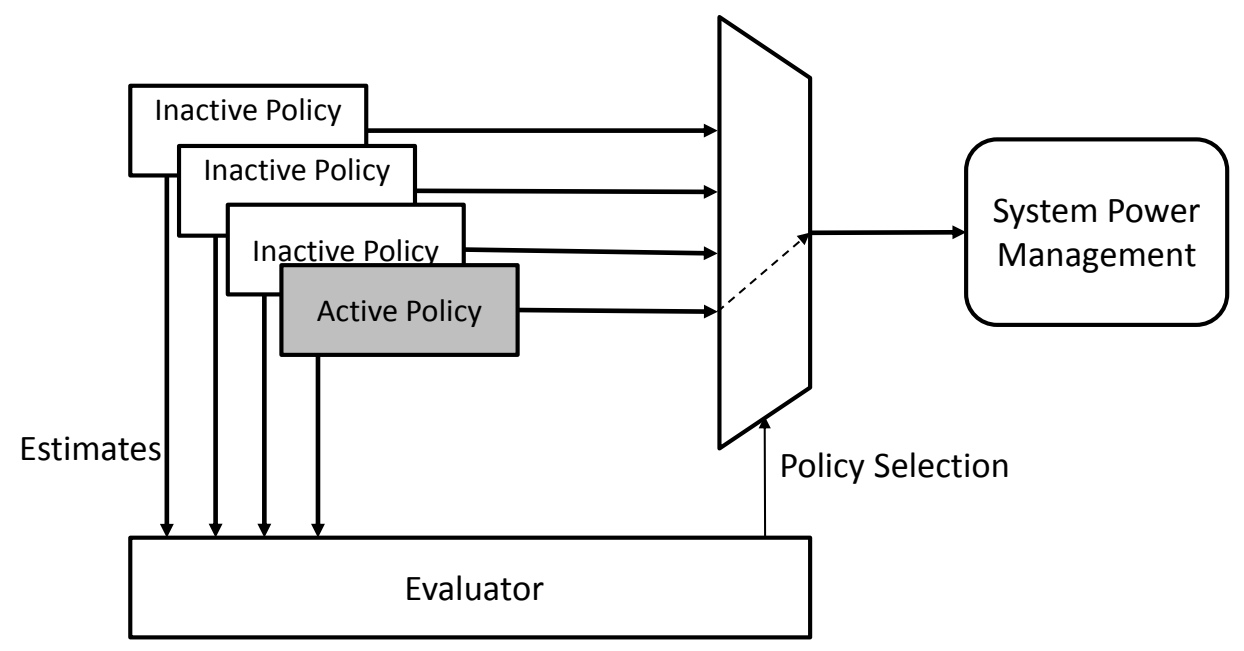

Fig. 4.7. Hybrid power management consists of a set of candidate power policies and an evaluator. Each candidate policy outperforms the others under different workloads. The candidate policies are evaluated based on a criteria of energy savings or delay during the policy evaluation. The best performing policy becomes active and manages the printer power for the next policy period. 


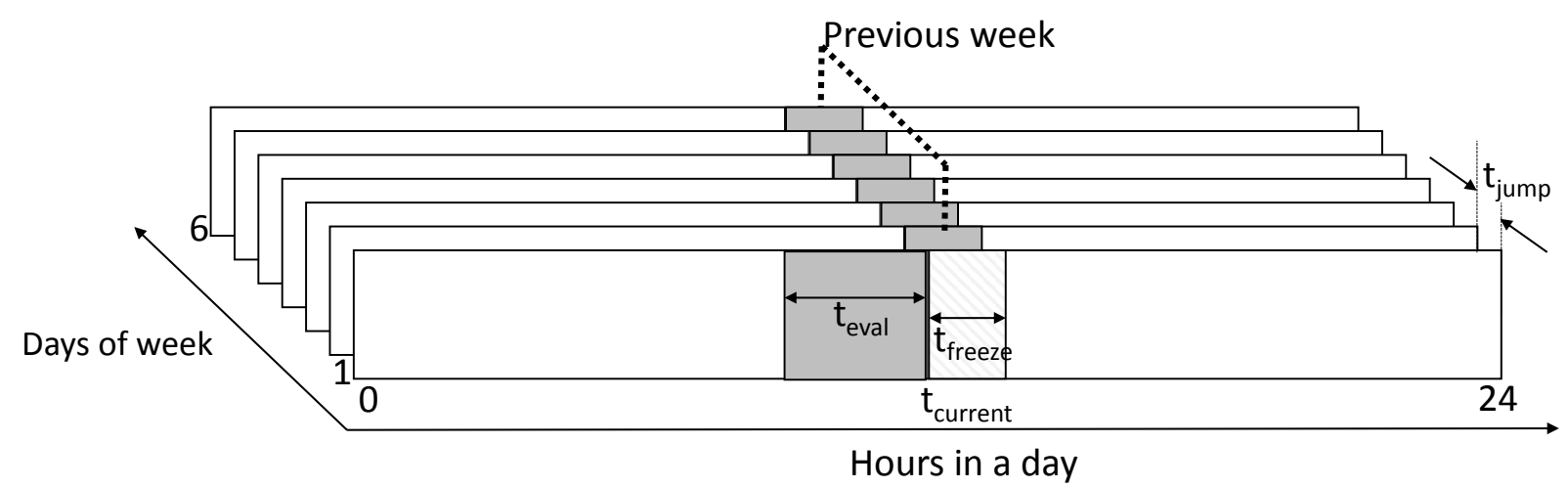

Fig. 4.8. Hybrid power manager with a policy evaluation window $\left(t_{\text {eval }}\right)$ and freeze window $\left(t_{\text {freeze }}\right)$. The best performing policy during the evaluation window is applied for the next freeze window. The evaluation window can be also set from the previous day or week. 


\section{EVALUATION AND RESULTS}

\subsection{Printer Parameter Measurement}

A multi-function printer is used to measure the parameters like wake-up delay, wake-up energy, average active power, average sleep power, and printer break-even time, as shown in Fig 5.1. The printer is connected to the main power source through a power meter. The power meter with a sensitivity of one milliwatts logs the power dissipated at an interval of one second. The power meter is connected to a network enabling readings to be logged remotely. The printer sleep function is enabled with a timeout of 30 minutes. Once the printer serves a request, it waits for a period of 30 minutes before transitioning to a low power sleep state. The standard ISO/IEC 24712:2006 color test pages are used for printing and measuring the parameters. The standard color test pages are used for the measurement of office equipment consumable yield. Fig 5.2 shows the power meter readings for a test page printing. From Fig 5.2, we observe that the printer consumes an average power of 12 Watts until the first request. As explained in Section 3.2, a printer consumes a nominal power during the sleep state, with most of the components turned off. There is a surge in power consumption with a request. The power surge reaches as high as 1000 Watts. This surge is due to the transient current drawn by the fuser heating the rod and other mechanical components like fan and motors. The power consumption stabilizes at around 600 Watts after the wakeup. The printer then enters the ready state consuming an average power of 70 Watts waiting for the future requests. The printer waits until a preset timeout (30 minutes) before entering the sleep state. The energy consumed and the elapsed time is measured for both the cases of printing from the sleep and ready state. The above experiment is repeated with one page, ten pages, and fifty pages per request. Table 5.1 lists the measured printer parameters. 


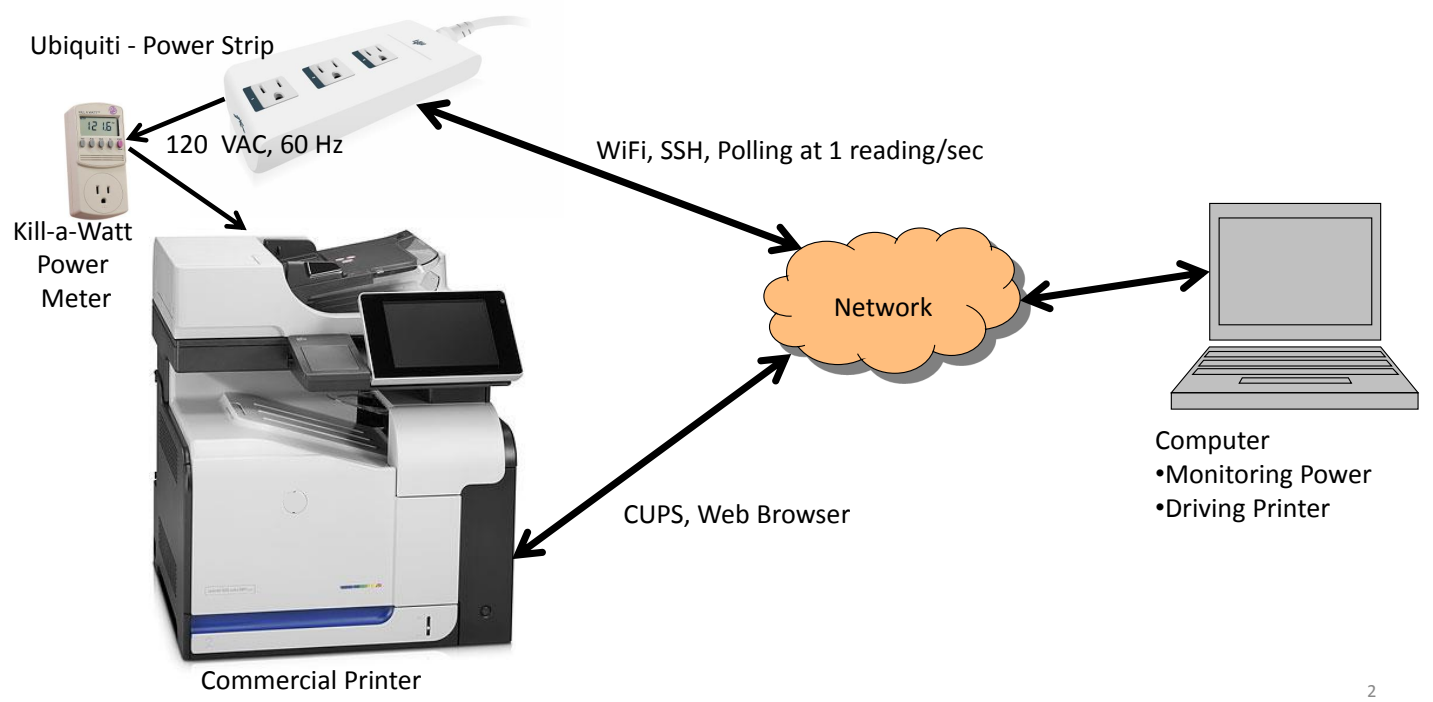

Fig. 5.1. Printer power measurement setup. The power meter and the printer are both connected to a network. A computer is used to control and access both the power meter and the printer. Common unix printing system (CUPS) standard is used to print to a network printer. Test pages are printed and the corresponding power readings are logged. The power meter refreshes the readings after each second. A $\log$ file is generated with the time-stamps and power readings.

Table 5.1.

Measured printer parameters

\begin{tabular}{|r|l|r|}
\hline SI.No & Parameter & Measured Values, Average \\
\hline \hline 1 & Wake-up Delay & 5 Seconds \\
\hline 2 & Wake-up Energy & 2909 Joules \\
\hline 3 & Active power & 70 Watts \\
\hline 4 & Sleep power & 12 Watts \\
\hline 5 & Break-even Time & 1.54 Minutes \\
\hline
\end{tabular}




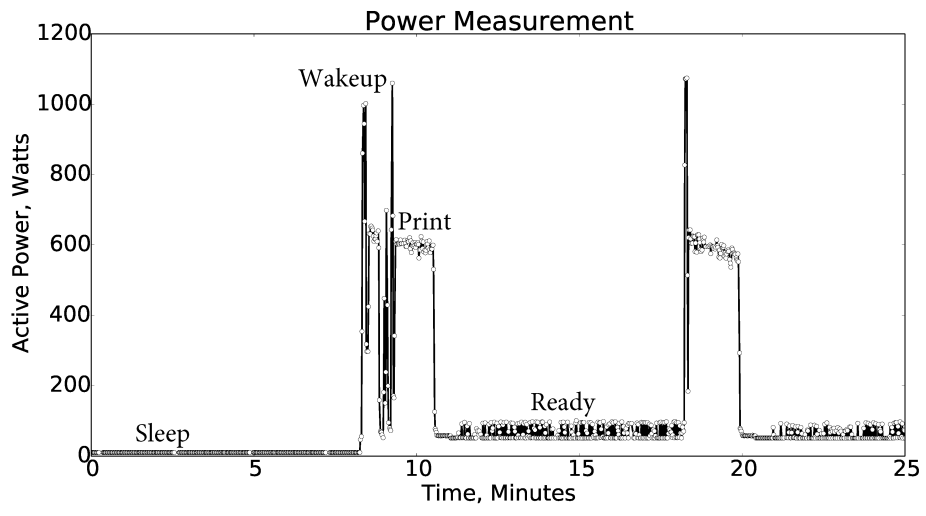

Fig. 5.2. Printer power measurement reading. A test page is printed while the printer is in (1) ready and (2) sleep state. Power readings are logged at each second. The printer starts in the sleep state consuming a low power. It serves a request consuming power at 650 Watts. The printer enters the ready state waiting for further requests, consuming an average idle power of 70 Watts. It consumes more power while printing from the sleep state than from the ready state. The printer takes more time serving a request from the sleep state. 


\subsection{Adaptive Multiphase Power Management}

We compare the multiphase policy's performance with several other policies: fixed one minute timeout, factory pre-set 30 minute timeout, and immediate sleeping. We perform simulations in MATLAB with the measured printer parameters and the printer traces. The simulation setup consists of the following: (1) The printer trace (2) The printer parameters (wakeup delay, wakeup energy, active power, and sleep power) (3) The MATLAB pattern search optimizer to compute multiphase parameters. The trace provides the sequence of inter-arrival times from the timestamps associated with each request used by the the printer model to compute the cumulative energy and delay. Based on the policy a corresponding timeout is applied for the simulation. The printer selects the states: idle, active, sleep and accumulates the energy and delay. At the end of the simulation, the average power and delay is computed. The pattern search optimizer is used to compute the multiphase policy's timeout and inter-arrival thresholds. Tables 5.2-5.3 provide the parameters computed from the pattern search for the quad-phase and the dual-phase policies. The pattern search is run multiple times to prevent the false convergence. The simulation is performed on the entire trace. But in actual implementation a sliding window can be adopted, whereby new set of parameters (thresholds and timeouts) are computed periodically from the available requests. For example, every day a new set of parameters can be computed from the past week requests. The quad-phase policy consists of four decreasing timeouts for phases ranging from the most busy to the least busy, and five increasing inter-arrival thresholds. Current simulation divides one hour into equal blocks based on the number of phases, and initializes the parameters assigning random values from each block. The search is performed 256 times and each time, the pattern search provides a set of parameters satisfying the delay constraint. The set of parameters that provide a low power is selected for the power management.

Fig 5.3 depicts the average power dissipated and the average delay per request encountered for different policies. The oracle policy provides the lower bound for 
power, as it knows the future and powers down appropriately. Fixed timeouts are poor at balancing both delay and power consumption. Immediate sleep and one minute timeout shuts down the printer very often causing increased delay. The 30 minute longer timeout rarely shuts down the printer and can serve a request immediately. However, it consumes the maximum power compared with other policies. As an example, for printer-5, the oracle policy expends on an average of 12.9 Watts with 3.3 seconds delay . The fixed timeout policies (30 min, one min, and immediate shutdown) consume 19.9, 13.6, and 13.2 Watts with $0.7 \mathrm{~s}, 3.7 \mathrm{~s}$, and $5 \mathrm{~s}$ delay respectively. The multiphase policy consumes 14 Watts with 3 seconds of delay. Thus, the multiphase policy achieves the performance goals with a marginal increase in power. The multiphase policy utilizes multiple timeouts to achieve the performance goals. The set of timeouts for a particular trace provides the best energy savings under a delay constraint. An oracle would choose large timeout during busy periods and immediate shutdown during reduced usage periods. The multiphase policy classifies the printer requests into finite activity phases. The printer incrementally moves to a relevant activity phase corresponding to the workload. Upon reaching the relevant activity phase, a corresponding timeout determined using pattern search is applied. The new set of thresholds and timeouts are computed again after a period with the consolidated requests.

\subsection{Hybrid Power Management}

The candidate power policies for the hybrid power management consists of the following:

1. Adaptive Multiphase Power Management

2. Fixed Timeout - 60 Seconds

3. Adaptive Timeout

4. 2-Competitive 
Table 5.2.

Quad-phase parameters using pattern search

\begin{tabular}{|c|r|r|r|r|r|r|r|}
\hline Printer & \multicolumn{6}{|c|}{ Timeouts (seconds) } & \multicolumn{3}{|c|}{$\begin{array}{l}\text { Inter-arrival } \\
\text { Thresholds } \\
\text { (seconds) }\end{array}$} \\
\hline & $T O_{0}$ & $T O_{1}$ & $T O_{2}$ & $T O_{3}$ & $I A_{0,1}$ & $I A_{1,2}$ & $I A_{2,3}$ \\
\hline \hline Printer-1 & 808 & 669 & 425 & 334 & 141 & 610 & 682 \\
\hline Printer-2 & 262 & 214 & 134 & 65 & 55 & 232 & 292 \\
\hline Printer-3 & 114 & 65 & 52 & 50 & 72 & 91 & 101 \\
\hline Printer-4 & 206.5 & 206 & 203 & 91 & 4 & 125 & 307 \\
\hline Printer-5 & 419 & 372 & 234 & 140 & 74 & 295 & 514 \\
\hline
\end{tabular}

Table 5.3.

Dual-Phase parameters using pattern search

\begin{tabular}{|c|r|r|r|}
\hline Printer & \multicolumn{2}{|l|}{$\begin{array}{l}\text { Timeouts } \\
\text { (seconds) }\end{array}$} & $\begin{array}{l}\text { Inter-arrival } \\
\text { Thresholds } \\
\text { (seconds) }\end{array}$ \\
\hline & $T O_{0}$ & $T O_{1}$ & $I A_{0,1}$ \\
\hline \hline Printer-1 & 227 & 137 & 1155 \\
\hline Printer-2 & 121 & 72 & 91 \\
\hline Printer-3 & 52 & 0 & 1256 \\
\hline Printer-4 & 141 & 73 & 76 \\
\hline Printer-5 & 240 & 103 & 115 \\
\hline
\end{tabular}






Fig. 5.3. Comparison of average power and average delay for different policies. The oracle policy saves the most energy as it knows the future. The factory preset fixed 30 minute timeout keeps the printer awake most of the time and hence less delay per request, but has the power consumption. Immediate shutdown has to wakeup for each request and hence has the delay per request. Multiphase policy balances delay with marginal penalty in power consumption.

A fixed timeout policy employs a single timeout irrespective of the workload. Fixed timeouts of one minute and break-even time are considered. The adaptive multiphase policy uses different phases each with an associated timeout. A set of inter-arrival thresholds are used to transition between phases based on the current inter-arrival time. Each multiphase parameter is computed from the past known requests as explained in Section 4.2. The current simulation uses a dual-phase policy with a delay constraint of less than 3 seconds. A sliding window approach is used for computing the parameters. The parameters are computed every day using the past week's requests. The adaptive power policy uses a dual timeout: 0 and break-even time, based on the inter-arrival time. The simulation setup consists of the following: (1) The printer trace (2) The printer parameters (3) The policy selection. The sequence of inter-arrival 
times, busy times, day of the week, and the type of request (print, copy, etc) are computed using the printer trace. Each of the policies are simulated using MATLAB with the measured printer parameters generating accumulated energy, delay, number of events, total trace duration, etc., which are used for comparisons and analysis. Table 5.4 lists the average power and Table 5.5 lists the average delay for each of the candidate power policies and printers. It is observed that no single policy is effective for all the printers. For example, the multiphase policy provides better energy savings for the printers- 2, 3, and 9. Whereas the adaptive policy provides energy savings for the printers- $1,5,6$, and 7 . Similarly, low delay is achieved using the multiphase policy for the printers- 1, 5, 7 and using 2-competitive policy for the printers- 3, 6, 9 .

The hybrid policy switches the candidate policies and provides better performance than the best performing individual policy. Current simulation uses the energy savings as the policy selection criteria. The hybrid policy evaluates the candidate policies' energy consumption during an evaluation window to select the best policy. The best policy performs the power management for a duration called policy freeze window. At the end of the policy freeze window, another evaluation is performed. From Table 5.4, we see that, the hybrid policy provides consistent energy savings than the best policy. The hybrid policy uses a combination of the individual policies to achieve better energy savings. Fig 5.4 shows the frequency of individual policy selection for printers at different offices. Depending upon the workload, each policy is selected to achieve better energy savings. For example, the hybrid policy selects the multiphase policy for $6.5 \%$, the adaptive policy for $63 \%$, and the fixed one minute timeout for $30 \%$ of the time for printer-2. From Table 5.8, we observe that there are 436 unique switches among power policies for one minute evaluation and freeze window. Thus, there are instances during the printer activity, wherein different policies provide better energy savings. Hence a single policy is ineffective for the entire workload and better energy savings can be achieved using a combination of policies. 
Table 5.4.

Average power (Watts) for the candidate power policies. No single policy provides low power for all the printers. The hybrid policy performs better than the best performing policy.

\begin{tabular}{|c|r|r|r|r|r|}
\hline Printer Trace & Multiphase & Fixed-60 & Adaptive & 2-Competitive & Hybrid \\
\hline \hline Printer-1 & 12.89 & 12.61 & 12.54 & 12.70 & 12.46 \\
\hline Printer-2 & 13.94 & 13.96 & 13.96 & 14.20 & 13.59 \\
\hline Printer-3 & 14.98 & 15.22 & 15.86 & 15.42 & 14.95 \\
\hline Printer-4 & 12.91 & 12.69 & 12.68 & 12.77 & 12.56 \\
\hline Printer-5 & 13.88 & 13.59 & 13.48 & 13.81 & 13.23 \\
\hline Printer-6 & 14.44 & 14.49 & 14.39 & 14.76 & 14.00 \\
\hline Printer-7 & 13.07 & 12.72 & 12.66 & 12.80 & 12.58 \\
\hline Printer-8 & 12.62 & 12.40 & 12.38 & 12.45 & 12.33 \\
\hline Printer-9 & 12.31 & 12.37 & 12.36 & 12.40 & 12.31 \\
\hline Printer-10 & 12.73 & 12.49 & 12.44 & 12.56 & 12.37 \\
\hline Printer-11 & 13.21 & 12.85 & 12.80 & 12.92 & 12.72 \\
\hline Printer-12 & 13.04 & 12.44 & 12.42 & 12.46 & 12.39 \\
\hline Printer-13 & 13.96 & 12.85 & 12.79 & 12.94 & 12.69 \\
\hline Printer-14 & 13.91 & 13.50 & 13.41 & 13.71 & 13.16 \\
\hline Printer-15 & 13.33 & 12.74 & 12.67 & 12.85 & 12.56 \\
\hline Printer-16 & 14.53 & 14.56 & 14.50 & 14.69 & 14.33 \\
\hline Printer-17 & 14.27 & 14.03 & 13.95 & 14.29 & 13.60 \\
\hline Printer-18 & 12.48 & 12.34 & 12.30 & 12.39 & 12.26 \\
\hline
\end{tabular}


Table 5.5.

Average delay (seconds) for the candidate power policies. No single policy provides low delay for all the printers.

\begin{tabular}{|c|r|r|r|r|r|}
\hline Printer Trace & Multiphase & Fixed-60 & Adaptive & 2-Competitive & Hybrid \\
\hline \hline Printer-1 & 2.84 & 3.26 & 3.47 & 3.08 & 3.14 \\
\hline Printer-2 & 3.07 & 3.05 & 3.39 & 2.80 & 2.87 \\
\hline Printer-3 & 2.78 & 2.23 & 2.44 & 1.96 & 2.50 \\
\hline Printer-4 & 2.80 & 2.97 & 3.23 & 2.77 & 2.81 \\
\hline Printer-5 & 3.19 & 3.44 & 3.76 & 3.22 & 3.30 \\
\hline Printer-6 & 2.94 & 2.82 & 2.98 & 2.50 & 2.53 \\
\hline Printer-7 & 3.15 & 3.47 & 3.76 & 3.26 & 3.38 \\
\hline Printer-8 & 2.67 & 3.19 & 3.47 & 3.04 & 3.03 \\
\hline Printer-9 & 1.35 & 1.15 & 1.25 & 1.08 & 1.12 \\
\hline Printer-10 & 3.13 & 3.65 & 3.95 & 3.47 & 3.47 \\
\hline Printer-11 & 2.74 & 3.28 & 3.57 & 3.09 & 3.09 \\
\hline Printer-12 & 2.67 & 3.37 & 3.73 & 3.21 & 3.19 \\
\hline Printer-13 & 2.77 & 3.55 & 3.85 & 3.41 & 3.45 \\
\hline Printer-14 & 2.96 & 3.31 & 3.60 & 3.13 & 3.03 \\
\hline Printer-15 & 2.97 & 3.58 & 3.80 & 3.41 & 3.46 \\
\hline Printer-16 & 3.44 & 3.36 & 3.72 & 3.15 & 3.25 \\
\hline Printer-17 & 3.10 & 3.38 & 3.69 & 3.09 & 3.18 \\
\hline Printer-18 & 3.08 & 3.54 & 3.75 & 3.30 & 3.31 \\
\hline
\end{tabular}


Table 5.6.

Average power (Watts) for Printer-2 with the hybrid policy using different evaluation and freeze windows

\begin{tabular}{|l|r|r|r|r|r|r|r|r|}
\hline $\begin{array}{l}\text { Evaluation } \\
\text { Window }\end{array}$ & \multicolumn{7}{|c|}{ Policy Freeze Window } \\
\hline & 1Min & 10 Min & 30Min & 1 Hour & 6 Hours & 12Hours & 1 Day & 1 Week \\
\hline \hline 1Min & 13.58 & 13.75 & 13.85 & 13.88 & 13.95 & 13.95 & 13.98 & 13.97 \\
\hline 1Hour & 13.84 & 13.90 & 13.92 & 13.93 & 13.93 & 13.93 & 13.98 & 13.97 \\
\hline 1Day & 13.92 & 13.94 & 13.96 & 13.95 & 13.97 & 13.98 & 13.98 & 13.95 \\
\hline 1Week & 13.95 & 13.96 & 13.96 & 13.95 & 13.97 & 13.97 & 13.96 & 13.95 \\
\hline
\end{tabular}

Table 5.7.

Average delay (seconds) for Printer-2 with the hybrid policy using different evaluation and freeze windows

\begin{tabular}{|l|r|r|r|r|r|r|r|r|}
\hline $\begin{array}{l}\text { Evaluation } \\
\text { Window }\end{array}$ & \multicolumn{9}{|c|}{ Policy Freeze Window } \\
\hline & 1Min & 10Min & 30Min & 1Hour & 6Hours & 12Hours & 1Day & 1 Week \\
\hline \hline 1Min & 2.87 & 3.07 & 3.18 & 3.20 & 3.26 & 3.26 & 3.32 & 3.34 \\
\hline 1Hour & 3.04 & 3.14 & 3.19 & 3.20 & 3.22 & 3.22 & 3.32 & 3.34 \\
\hline 1Day & 3.17 & 3.18 & 3.21 & 3.20 & 3.23 & 3.24 & 3.30 & 3.12 \\
\hline 1Week & 3.14 & 3.17 & 3.17 & 3.19 & 3.22 & 3.22 & 3.17 & 3.12 \\
\hline
\end{tabular}


Table 5.8 .

Policy switch frequency for Printer-2 with the hybrid policy using different evaluation and freeze windows

\begin{tabular}{|l|r|r|r|r|r|r|r|r|}
\hline $\begin{array}{l}\text { Evaluation } \\
\text { Window }\end{array}$ & \multicolumn{10}{|c|}{ Policy Freeze Window } \\
\hline & 1 Min & 10 Min & 30 Min & 1 Hour & 6 Hours & 12 Hours & 1 Day & 1 Week \\
\hline \hline 1 Min & 436 & 224 & 117 & 70 & 21 & 18 & 9 & 2 \\
\hline 1 Hour & 278 & 191 & 131 & 105 & 25 & 23 & 9 & 2 \\
\hline 1 Day & 119 & 86 & 66 & 44 & 30 & 30 & 20 & 4 \\
\hline 1 Week & 45 & 35 & 23 & 17 & 15 & 15 & 12 & 4 \\
\hline
\end{tabular}




\subsection{Evaluation Window Selection}

We next perform policy selection simulations on printers from 18 different offices to find the evaluation and freeze windows that achieve the best energy savings. As explained in the previous section, the hybrid policy with four different policies is used for the simulation. We simulate the hybrid policy with different sets of time periods for the evaluation and freeze window. The best policy based on the energy saving criteria during an evaluation window is selected and activated for the next freeze window. Following evaluation windows are considered for the simulation: 1 Minute, 1 Hour, 1 Day, and 1 Week. Following freeze windows are considered: 1, 10, 30 Minutes, 1, 6, 12 Hours, 1 Day, and 1 Week.

Fig 5.5 shows the average power for different evaluation and freeze windows. We see that using a short evaluation and freeze window, better energy savings can be achieved. For the current simulation, the hybrid policy using 1 minute evaluation and freeze window provides the best energy savings compared with the other combinations. The hybrid policy using a shorter evaluation window relies less on the distant past requests and hence the selected policy is more suited for the current workload. We see that using a longer evaluation window, the energy consumption approaches that of the best individual policy. A shorter freeze window, enables the hybrid policy to update and switch policies more often based on the workload variation. However using a longer freeze window, the hybrid policy is restricted in updating the policies with the workload variations. Also any wrong selection of policy would take a longer time to be corrected and hence energy is wasted. Tables 5.6-5.8 list the following results of the simulation for printer-2: average power, average delay, and policy switch frequency. Thus, the hybrid policy using short-term memory and updated frequently provides the best energy savings. 




Fig. 5.4. Histogram of power management policies selected by the hybrid policy. This shows that the best policies varies with the underlying requests.



Fig. 5.5. Average power in Watts for Printer-2 with hybrid policy using different evaluation and freeze windows 


\subsection{Week Analysis}

We next perform simulations with scheduling information from the past six days and the previous week for policy selections. We are motivated by the printer request pattern, as explained in Section 4.1. Most of the printer traces display distinct request patterns - with significant requests during weekdays followed by limited requests during weekends. Similarly, requests are frequent during working hours as compared with non-working hours. We perform simulations to verify any energy savings using this scheduling information. We perform simulations, selecting the best policy from the previous six days and the previous week. Tables 5.9-5.10 list the simulation results for the hybrid policy with scheduling information from the previous six days and the previous week. With the previous six days schedule, we evaluate the candidate power policies from the previous six days at the current time. For example, if the current time is $11 \mathrm{AM}$, the candidate policies are evaluated from the previous six days at exactly 11 AM. The policy that provides the best energy savings for most number of days is selected. In the previous week schedule, best policy from the previous week is selected at the current time. From Tables 5.9-5.10, we observe that the regular hybrid policy without using any scheduling information performs better than the policies using scheduling information. The current workload cannot be characterized using the past days' or the previous week information, although we observe a pattern among printer requests. The current workload is different from the workload that existed during previous days or the previous week. Hence, scheduling information from the past requests (previous days or week) does not provide consistent energy savings. 
Table 5.9.

Average power in Watts for the previous six day schedule. The hybrid policy using a short-term memory of past requests and frequent updates performs better than the policies using scheduling information from the previous six days.

\begin{tabular}{|l|l|l|l|l|l|l|l|l|}
\hline $\begin{array}{l}\text { Printer } \\
\text { trace }\end{array}$ & 1 Min & 10 Min & 30 Min & 1 Hour & 6 Hour & 12 Hour & 1 Day & $\begin{array}{l}\text { Hyb- } \\
\text { rid }\end{array}$ \\
\hline \hline Printer-1 & 12.56 & 12.56 & 12.55 & 12.55 & 12.55 & 12.55 & 12.54 & 12.46 \\
\hline Printer-2 & 13.98 & 13.98 & 13.98 & 14.00 & 13.99 & 13.99 & 13.98 & 13.59 \\
\hline Printer-3 & 15.32 & 15.30 & 15.28 & 15.23 & 15.12 & 15.11 & 15.11 & 14.95 \\
\hline Printer-4 & 12.68 & 12.68 & 12.68 & 12.68 & 12.68 & 12.68 & 12.68 & 12.56 \\
\hline Printer-5 & 13.50 & 13.50 & 13.52 & 13.52 & 13.51 & 13.51 & 13.48 & 13.23 \\
\hline Printer-6 & 14.39 & 14.38 & 14.36 & 14.37 & 14.37 & 14.38 & 14.40 & 14.00 \\
\hline Printer-7 & 12.67 & 12.67 & 12.67 & 12.67 & 12.67 & 12.67 & 12.67 & 12.58 \\
\hline Printer-8 & 12.41 & 12.41 & 12.41 & 12.41 & 12.41 & 12.41 & 12.39 & 12.33 \\
\hline Printer-9 & 12.36 & 12.36 & 12.36 & 12.36 & 12.35 & 12.35 & 12.33 & 12.31 \\
\hline Printer-10 & 12.44 & 12.44 & 12.44 & 12.44 & 12.44 & 12.44 & 12.44 & 12.37 \\
\hline Printer-11 & 12.83 & 12.83 & 12.83 & 12.83 & 12.82 & 12.82 & 12.83 & 12.72 \\
\hline Printer-12 & 12.42 & 12.42 & 12.42 & 12.42 & 12.42 & 12.42 & 12.43 & 12.39 \\
\hline Printer-13 & 12.79 & 12.79 & 12.79 & 12.79 & 12.79 & 12.79 & 12.79 & 12.69 \\
\hline Printer-14 & 13.46 & 13.46 & 13.46 & 13.46 & 13.45 & 13.46 & 13.43 & 13.16 \\
\hline Printer-15 & 12.68 & 12.68 & 12.69 & 12.68 & 12.68 & 12.68 & 12.69 & 12.56 \\
\hline Printer-16 & 14.47 & 14.48 & 14.48 & 14.47 & 14.47 & 14.47 & 14.47 & 14.33 \\
\hline Printer-17 & 14.00 & 13.99 & 13.99 & 13.98 & 14.02 & 14.00 & 13.97 & 13.60 \\
\hline Printer-18 & 12.31 & 12.31 & 12.31 & 12.31 & 12.31 & 12.31 & 12.31 & 12.26 \\
\hline
\end{tabular}


Table 5.10.

Average power in Watts for the previous week schedule. The hybrid policy without using any scheduling information from the past week performs consistently better than the policies using scheduling information.

\begin{tabular}{|l|l|l|l|l|l|l|l|l|l|}
\hline $\begin{array}{l}\text { Printer } \\
\text { Trace }\end{array}$ & 1 Min & 10 Min & 30 Min & 1 Hour & 6 Hour & 12 Hour & 1 Day & 1 Week & $\begin{array}{l}\text { Hyb- } \\
\text { rid }\end{array}$ \\
\hline \hline Printer-1 & 12.59 & 12.59 & 12.59 & 12.59 & 12.58 & 12.58 & 12.57 & 12.54 & 12.46 \\
\hline Printer-2 & 14.02 & 14.00 & 13.98 & 13.98 & 13.97 & 13.97 & 13.96 & 13.95 & 13.59 \\
\hline Printer-3 & 15.33 & 15.29 & 15.28 & 15.26 & 15.21 & 15.20 & 15.11 & 14.98 & 14.95 \\
\hline Printer-4 & 12.69 & 12.68 & 12.68 & 12.67 & 12.67 & 12.67 & 12.67 & 12.67 & 12.56 \\
\hline Printer-5 & 13.53 & 13.52 & 13.53 & 13.55 & 13.55 & 13.55 & 13.56 & 13.48 & 13.23 \\
\hline Printer-6 & 14.47 & 14.44 & 14.46 & 14.42 & 14.38 & 14.38 & 14.39 & 14.39 & 14.00 \\
\hline Printer-7 & 12.72 & 12.71 & 12.71 & 12.71 & 12.69 & 12.69 & 12.69 & 12.68 & 12.58 \\
\hline Printer-8 & 12.49 & 12.49 & 12.49 & 12.49 & 12.49 & 12.49 & 12.48 & 12.48 & 12.33 \\
\hline Printer-9 & 12.36 & 12.36 & 12.35 & 12.35 & 12.34 & 12.34 & 12.33 & 12.32 & 12.31 \\
\hline Printer-10 & 12.45 & 12.45 & 12.45 & 12.45 & 12.45 & 12.45 & 12.45 & 12.44 & 12.37 \\
\hline Printer-11 & 12.85 & 12.84 & 12.84 & 12.83 & 12.83 & 12.83 & 12.83 & 12.80 & 12.72 \\
\hline Printer-12 & 12.43 & 12.43 & 12.43 & 12.43 & 12.43 & 12.43 & 12.43 & 12.42 & 12.39 \\
\hline Printer-13 & 12.82 & 12.81 & 12.81 & 12.82 & 12.81 & 12.81 & 12.80 & 12.79 & 12.69 \\
\hline Printer-14 & 13.45 & 13.45 & 13.44 & 13.44 & 13.44 & 13.44 & 13.43 & 13.43 & 13.16 \\
\hline Printer-15 & 12.70 & 12.70 & 12.71 & 12.71 & 12.70 & 12.70 & 12.71 & 12.67 & 12.56 \\
\hline Printer-16 & 14.50 & 14.50 & 14.50 & 14.50 & 14.49 & 14.49 & 14.49 & 14.48 & 14.33 \\
\hline Printer-17 & 14.02 & 14.01 & 14.01 & 14.01 & 14.05 & 14.03 & 14.01 & 14.00 & 13.60 \\
\hline Printer-18 & 12.32 & 12.32 & 12.32 & 12.32 & 12.31 & 12.31 & 12.31 & 12.31 & 12.26 \\
\hline
\end{tabular}




\section{SUMMARY}

In this thesis, two power management policies are presented for office equipment: adaptive multiphase policy and hybrid policy. The multiphase policy compartmentalizes the printer workload into finite phases. The printer transitions to a relevant phase, based on the current workload and an appropriate timeout is applied balancing power and delay. The hybrid policy selects the best performing policy to perform system power management. We arrive at the appropriate policy evaluation and freeze windows and show that frequent updates with short-term memory provides the best performance. We also show that, policies relying on scheduling information do not provide consistent energy savings. We perform the simulations measuring the printer parameters from a commercial printer and using the request traces from printers with contrasting usages. 
REFERENCES 


\section{REFERENCES}

[1] K. Kawamoto, Y. Shimoda, and M. Mizuno, "Energy saving potential of office equipment power management," Energy and Buildings, vol. 36, no. 9, pp. 915923, 2004.

[2] Energy Star Program Requirements for Imaging Equipment Partner Commitments, 2014.

[3] Blue Angel - Basic Criteria for Award of the Environmental Label - Office Equipment with Printing Function, 2013.

[4] Nordic Ecolabelling of Imaging equipment, 2013.

[5] A. K. Meier and B. LeBot, "One watt initiative: A global effort to reduce leaking electricity," Lawrence Berkeley National Laboratory, 1999.

[6] Y.-H. Lu, E.-Y. Chung, T. Simunic, L. Benini, and G. De Micheli, "Quantitative comparison of power management algorithms," in Design, Automation and Test in Europe Conference and Exhibition. Proceedings. IEEE, 2000, pp. 20-26.

[7] L. Benini, A. Bogliolo, and G. De Micheli, "A survey of design techniques for system-level dynamic power management," Very Large Scale Integration (VLSI) Systems, IEEE Transactions on, vol. 8, no. 3, pp. 299-316, 2000.

[8] H. Shen, Y. Tan, J. Lu, Q. Wu, and Q. Qiu, "Achieving autonomous power management using reinforcement learning," ACM Transactions on Design Automation of Electronic Systems (TODAES), vol. 18, no. 2, p. 24, 2013.

[9] G. Dhiman and T. S. Rosing, "Dynamic power management using machine learning," in Proceedings of the 2006 IEEE/ACM international conference on Computer-aided design. ACM, pp. 747-754.

[10] Y. Wang, Q. Xie, A. Ammari, and M. Pedram, "Deriving a near-optimal power management policy using model-free reinforcement learning and bayesian classification," in Proceedings of the 48th Design Automation Conference. ACM, 2011, pp. 41-46.

[11] G. Dhiman and T. S. Rosing., "System-level power management using online learning." in IEEE Transactions on Computer-Aided Design of Integrated Circuits and Systems. IEEE, 2009, pp. 676-689.

[12] N. Pettis and Y.-H. Lu, "A homogeneous architecture for power policy integration in operating systems," IEEE Transactions on Computers, vol. 58, no. 7, pp. 945-955, 2009.

[13] V. Ciriza, C. R. Dance, and L. Donini, "Printer time-out," Patent US 8230 248, Jul. 24, 2012. 
[14] V. Ciriza, L. Donini, J.-B. Durand, S. Girard et al., "A statistical model for optimizing power consumption of printers," in 40èmes Journées de Statistique, 2008.

[15] L. Benini and G. d. Micheli, "System-level power optimization: techniques and tools," ACM Transactions on Design Automation of Electronic Systems (TO$D A E S)$, vol. 5, no. 2, pp. 115-192, 2000.

[16] S. Irani, G. Singh, S. K. Shukla, and R. K. Gupta, "An overview of the competitive and adversarial approaches to designing dynamic power management strategies," Very Large Scale Integration (VLSI) Systems, IEEE Transactions on, vol. 13, no. 12, pp. 1349-1361, 2005.

[17] F. Douglis, P. Krishnan, B. Bershad et al., "Adaptive disk spin-down policies for mobile computers," Computing Systems, vol. 8, no. 4, pp. 381-413, 1995.

[18] D. Ramanathan and R. Gupta, "System level online power management algorithms," in Design, Automation and Test in Europe Conference and Exhibition 2000. Proceedings. IEEE, 2000, pp. 606-611.

[19] H.-C. Shih and K. Wang, "An adaptive hybrid dynamic power management algorithm for mobile devices," Computer Networks, vol. 56, no. 2, pp. 548-565, 2012.

[20] R. Golding, P. Bosch, and J. Wilkes, "Idleness is not sloth," USENIX Winter Conference, pp. 201-212, 1995.

[21] C.-H. Hwang and A. C.-H. Wu, "A predictive system shutdown method for energy saving of event-driven computation," ACM Transactions on Design Automation of Electronic Systems (TODAES), vol. 5, no. 2, pp. 226-241, 2000.

[22] M. B. Srivastava, A. P. Chandrakasan, and R. W. Brodersen, "Predictive system shutdown and other architectural techniques for energy efficient programmable computation," Very Large Scale Integration (VLSI) Systems, IEEE Transactions on, vol. 4, no. 1, pp. 42-55, 1996.

[23] E.-Y. Chung, L. Benini, A. Bogliolo, Y.-H. Lu, and G. De Micheli, "Dynamic power management for nonstationary service requests," Computers, IEEE Transactions on, vol. 51, no. 11, pp. 1345-1361, 2002.

[24] Q. Qiu and M. Pedram, "Dynamic power management based on continuoustime markov decision processes," in Proceedings of the 36th annual ACM/IEEE Design Automation Conference. ACM, 1999, pp. 555-561.

[25] G. Theocharous, S. Mannor, N. Shah, P. Gandhi, B. Kveton, S. Siddiqi, and C.-H. Yu, "Machine learning for adaptive power management," Intel Technology Journal, vol. 10, pp. 299-312, 2006.

[26] U. A. Khan and B. Rinner, "Online learning of timeout policies for dynamic power management," ACM Transactions on Embedded Computing Systems (TECS), vol. 13, no. 4, p. 96, 2014.

[27] G. Tesauro, R. Das, H. Chan, J. Kephart, D. Levine, F. Rawson, and C. Lefurgy, "Managing power consumption and performance of computing systems using reinforcement learning," in Advances in Neural Information Processing Systems, 2007, pp. 1497-1504. 
[28] Y. Tan, W. Liu, and Q. Qiu, "Adaptive power management using reinforcement learning," in Proceedings of the 2009 International Conference on ComputerAided Design. ACM, pp. 461-467.

[29] A. R. Karlin, M. S. Manasse, L. A. McGeoch, and S. Owicki, "Competitive randomized algorithms for nonuniform problems," Algorithmica, vol. 11, no. 6, pp. 542-571, 1994.

[30] S. Irani and K. R. Pruhs, "Algorithmic problems in power management," $A C M$ SIGACT News, vol. 36, no. 2, pp. 63-76, 2005.

[31] Y. Guo, R. Li, G. Poulton, and A. Zeman, "A simulator for self-adaptive energy demand management," in Self-Adaptive and Self-Organizing Systems. SASO. Second IEEE International Conference on. IEEE, 2008, pp. 64-73.

[32] G. Samorodnitsky, "Long memory and self-similar processes," in Annales-Faculte des Sciences Toulouse Mathematiques, vol. 15, no. 1. Citeseer, 2006, p. 107.

[33] B. R. Larson, "Managing a power state for a peripheral," Patent US 7904739 , Mar. 8, 2011.

[34] J.-B. Durand, S. Girard, V. Ciriza, and L. Donini, "Optimization of power consumption and device availability based on point process modelling of the request sequence," Journal of the Royal Statistical Society: Series C (Applied Statistics), vol. 62, no. 2, pp. 151-165, Mar. 2013.

[35] D. P. Helmbold, D. D. Long, T. L. Sconyers, and B. Sherrod, "Adaptive disk spindown for mobile computers," Mobile Networks and Applications, vol. 5, no. 4, pp. 285-297, 2000.

[36] Hewlett-Packard, HP Color LaserJet CM3530 MFP Series Service Manual. Hewlett-Packard, 2008.

[37] J. Knoder, "Best laser printer reviews and comparisons," http://printers.toptenreviews.com/laser/, 2015.

[38] T. G. Kolda, R. M. Lewis, and V. Torczon, "Optimization by direct search: New perspectives on some classical and modern methods," SIAM review, vol. 45, no. 3, pp. 385-482, 2003.

[39] I. The MathWorks, "Matlab global optimization toolbox - patternsearch," http://www.mathworks.com/help/gads/patternsearch.html, 2015. 